\title{
On the piecewise smoothness of entropy solutions to scalar conservation laws for a large class of initial data
}

\author{
Tao Tang* Jinghua Wang ${ }^{\dagger} \quad$ Yinchuan Zhao ${ }^{\ddagger}$
}

\begin{abstract}
In this paper, we prove that if the initial data do not belong to a certain subset of $C^{k}$, which is hitherto smallest in the sense of inclusion relation of sets, then the solutions of scalar conservation laws are piecewise smooth. In particular, our initial data allow centered compression waves, which is the case not covered by Dafermos (1974) and Schaeffer (1973). More precisely, we are concerned with the structure of the solutions in the neighborhoods of the points at which only a $C^{k+1}$ shock generates, while there can be infinite number of intervals, the characteristics from each of these intervals will meet at a point in any small neighborhood. We give sufficient and almost necessary conditions of the initial data for a degenerate point at which a $C^{k+1}$ shock generates. It is also shown that there are finitely many shocks for smooth initial data (in the Schwartz space) except a certain subset of $\mathscr{S}(\mathbb{R})$ of the first category. It should be pointed out that this subset is smaller than those used in previous works. We point out that Thom's theory of catastrophes [10], which plays a key role in Schaeffer [9], can not be used to analyze the larger class of initial data considered in this work.
\end{abstract}

\section{Introduction}

Consider the Cauchy problem for the scalar hyperbolic conservation law:

$$
\left\{\begin{aligned}
u_{t}+f(u)_{x}=0 & & \text { in } \mathbb{R} \times(0, \infty) \\
u=\phi & & \text { on } \mathbb{R} \times\{t=0\},
\end{aligned}\right.
$$

where $f$ is $C^{k+1}$ smooth and uniformly convex, i.e., $\partial^{2} f / \partial u^{2} \geq \epsilon>0$, the initial data are $C^{k}$ smooth and bounded, with $3 \leq k \leq \infty$. In general, the problem (1.1) does not admit a global smooth solution even if the initial data are smooth, but for arbitrary bounded measurable initial data a unique global weak solution does exist. The structure of entropy solution has been studied by many authors, e.g., Chen-Zhang [1], Dafermos [2], Lax [5], Li-Wang [6, 7], Oleinik [8], Schaeffer [9], and Tadmor \& Tassa [11].

\footnotetext{
${ }^{*}$ Department of Mathematics, Hong Kong Baptist University, Kowloon Tong Hong Kong \& Institute of Computational Mathematics, Chinese Academy of Sciences, Beijing, China. Email: ttang@math.hkbu.edu.hk.

${ }^{\dagger}$ Institute of Systems Sciences, Academy of Mathematics and System Sciences, The Chinese Academy of Sciences, Beijing 100080, P. R. China. Email: jwang@amss.ac.cn.

${ }^{\ddagger}$ Institute of Computational Mathematics, Academy of Mathematics and System Sciences, The Chinese Academy of Sciences, Beijing 100080, P. R. China. Email: zyc747250@hotmail.com.
} 
The main results of this work will be obtained by using the minimizing process of $F(x, t, u)$ introduced by Lax [5]: For each initial function $\phi$ we define a function $H \times \mathbb{R}$ :

$$
F(x, t, u)=\operatorname{tg}(u)+\Phi(x-t a(u))
$$

where $H=\mathbb{R} \times(0, \infty), a(u)=f^{\prime}(u)$,

$$
g(u)=u a(u)-f(u), \quad \Phi(y)=\int_{0}^{y} \phi(x) d x .
$$

Lax has proved that for almost all $(x, t)$ there exists a unique value of $u$ which minimizes $F(x, t, \bullet)$, and $u(x, t)$, the function defined(almost everywhere) to equal the value of $u$ which minimizes $F(x, t, \bullet)$, is in fact the solution of (1.1). It follows from the convexity hypothesis and the fact that the initial function is bounded that $F(x, t, u) \rightarrow+\infty$ as $u \rightarrow \pm \infty$. Therefore, $F(x, t, \bullet)$ always has a minimum, and a minimizing value $u$ must be a critical point of $F$, a solution of the equation

$$
(\partial F / \partial u)(x, t, u)=0 .
$$

Note that

$$
(\partial F / \partial u)(x, t, u)=t a^{\prime}(u)\{u-\phi(x-t a(u))\}
$$

and neither of the factors outside the brackets vanishes. It can be verified that the differential of $\partial F / \partial u$ never vanishes when $\partial F / \partial u=0$, so (1.3) defines a smooth surface $S$ in $H \times \mathbb{R}$. we record here the following relations that will be needed below:

$$
\begin{array}{ll}
(\partial F / \partial x)(x, t, u)=u & \text { on } S \\
(\partial F / \partial t)(x, t, u)=-f(u) & \text { on } S .
\end{array}
$$

Let us introduce some notations and definitions. First let

$$
\begin{aligned}
& L_{1}=\left\{x \in \mathbb{R} \mid(a(\phi(x)))^{\prime}<0,(a(\phi(x)))^{\prime \prime}=0,(a(\phi(x)))^{\prime \prime \prime}=0\right\} \\
& L_{2}=\left\{x \in \mathbb{R} \mid(a(\phi(x)))^{\prime}<0,(a(\phi(x)))^{\prime \prime}=0, \cdots,(a(\phi(x)))^{(k)}=0, k \geq 3\right\}
\end{aligned}
$$

and

$$
\begin{aligned}
L_{3}= & \left\{x \in \mathbb{R} \mid(a(\phi(x)))^{\prime}<0,\left(a(\phi(x))^{\prime \prime}=0, \cdots,(a(\phi(x)))^{(k)}=0,(k \geq 3) \&\right.\right. \\
& \text { there exists a point } \xi \in(x, x+\delta) \text { such that }(a(\phi(\xi)))^{\prime \prime}<0 \text { or } \\
& \text { a point } \left.\eta \in(x-\delta, x) \text { such that }(a(\phi(\eta)))^{\prime \prime}>0 \text { for any } \delta>0\right\} .
\end{aligned}
$$

Remark 1.1 It can be verified that $L_{2}$ is a proper subset of $L_{1}$ when $k>3$ and $L_{3}$ is a proper subset of $L_{2}$ when $k \geq 3$.

Definition 1.1 Let $u_{0}$ be a minimizing value for $F\left(x_{0}, t_{0}, \bullet\right), u_{0}$ is called non-degenerate (degenerate) if $F_{u u}\left(x_{0}, t_{0}, u_{0}\right) \neq 0(=0)$. 
Definition 1.2 The solution $u(x, t)$ is said to be $C^{k}$ piecewise smooth if every bounded subset of $(-\infty, \infty) \times[0, \infty)$ intersects at most a finite number of shocks, every shock is piecewise $C^{k+1}$ smooth curve, and $u(x, t)$ is $C^{k}$ smooth on the complement of the shock set.

To summarize some previous result, we will first give some definitions. Let

$U=\left\{(x, t): \exists\right.$ unique minimizing value for $F(x, t, \bullet)$, on which $\left.F_{u u} \neq 0\right\}$,

$\Gamma_{1}=\left\{(x, t): \exists\right.$ two minimizing values for $F(x, t, \bullet)$, on which $\left.F_{u u} \neq 0\right\}$,

$\Gamma_{0}^{(c)}=\left\{(x, t): \exists\right.$ three minimizing values for $F(x, t, \bullet)$, on which $\left.F_{u u} \neq 0\right\}$,

$\Gamma_{0}^{(f)}=\left\{(x, t): \exists\right.$ unique minimizing value for $F(x, t, \bullet)$, on which $\left.F_{u u}=0, F_{u}^{(4)} \neq 0\right\}$.

Moreover, let $\mathscr{M}_{(x, t)}$ be the set consisting of all the minimizing values for $F(x, t, \bullet)$, and

$\bar{\Gamma}_{1}=\left\{(x, t): \exists\right.$ two connected components of $\left.\mathscr{M}_{(x, t)}\right\}$,

$\bar{\Gamma}_{0}^{(f)}=\left\{(x, t): \exists\right.$ unique connected component $[\alpha, \beta]$ of $\mathscr{M}_{(x, t)}$, and $\left.F_{u u}(x, t, \alpha)=0\right\}$,

$\bar{\Gamma}_{0}^{(c)}=\left\{(x, t): \exists n\right.$ connected components of $\mathscr{M}_{(x, t)}$, where $\left.n \geq 3\right\}$.

It is shown by Schaeffer [9] that $U$ is an open subset of $H=\mathbb{R} \times(0, \infty)$, on which the solution $u(x, t)$ is smooth; $\Gamma_{1}$ is a union of smooth curves across which the minimizing function has a jump discontinuity and that $\Gamma_{0}^{(c)}$ and $\Gamma_{0}^{(f)}$ consist of isolated points at which the curves in $\Gamma_{1}$ begin to collide. It is obvious that $\Gamma_{0}^{(f)}$ is a proper subset of $\bar{\Gamma}_{0}^{(f)}, \Gamma_{1}$ is a proper subset of $\bar{\Gamma}_{1}$ and $\Gamma_{0}^{(c)}$ is a proper subset of $\bar{\Gamma}_{0}^{(c)}$. It has been proved by Schaeffer [9] that the solutions of (1.1) are piecewise smooth and the total number of shocks is finite for smooth initial data (in the Schwartz space) except in a certain subset of $\mathscr{S}(\mathbb{R})$ of the first category. In other words, there is a set $\Omega \subset \mathscr{S}(\mathbb{R})$ of first category such that for $\phi \in \mathscr{S}(\mathbb{R}) \sim \Omega$

$$
H=U \cup \Gamma_{1} \cup \Gamma_{0}^{(c)} \cup \Gamma_{0}^{(f)} .
$$

Lemma 1.4 in [9] is very important, which shows that any point $\left(x_{0}, t_{0}\right) \in \Gamma_{0}^{(f)}$ has a neighborhood $\Theta$ such that $\Gamma_{1} \cap \Theta$ consists of a half-curve originating at $\left(x_{0}, t_{0}\right)$. The minimizing function is smooth on $\Theta^{\prime} \sim \Gamma_{1}$, where $\Theta^{\prime}=\Theta \sim\left\{\left(x_{0}, t_{0}\right)\right\}$. The proof is an adaption of standard techniques from the theory of singularities of differentiable mappings, especially Thom's theory of catastrophes [10]. Schaeffer made a serious attempt to make this material accessible to analysts, including reproving the so called universal unfolding of the Riemann-Hugoniot catastrophe, one of the seven elementary catastrophes of Thom [10]. Section 2 in [9] is devoted to prove the unfolding theorem for the Riemann-Hugoniot catastrophe.

It is naturally to ask if the conclusions of Lemma 1.4 in Schaeffer [9] are true when

$$
F_{u}\left(x_{0}, t_{0}, u_{0}\right)=0, \quad F_{u u}\left(x_{0}, t_{0}, u_{0}\right)=0, \cdots, F_{u}^{(2 n-1)}\left(x_{0}, t_{0}, u_{0}\right)=0, F_{u}^{(2 n)}\left(x_{0}, t_{0}, u_{0}\right)>0
$$

where $n \geq 3$ is some integer; or even $F_{u}^{(m)}\left(x_{0}, t_{0}, u_{0}\right)=0,(m=1,2, \cdots)$. Unfortunately, the unfolding theorem for the Riemann-Hugoniot catastrophe is not applicable in this case since $F(x, t, u)$ is unstable as an unfolding of codimension two of $F\left(x_{0}, t_{0}, u\right)$ when

$$
F_{u}\left(x_{0}, t_{0}, u_{0}\right)=0, \quad F_{u u}\left(x_{0}, t_{0}, u_{0}\right)=0, \quad F_{u}^{(3)}\left(x_{0}, t_{0}, u_{0}\right), \quad F_{u}^{(4)}\left(x_{0}, t_{0}, u_{0}\right)=0 .
$$


One of the main purposes of this work is to show that the conclusions of Lemma 1.4 in [9] are true under the conditions $L_{3}=\emptyset$, which is more general than the condition (1.9). The method used in our proof is different from the one used by Schaeffer, which is elementary but technical.

It is shown by $\mathrm{Li}$ and Wang [6] that there is a set $\Omega_{1} \subset \Omega \subset \mathscr{S}(\mathbb{R})$ of first category such that for $\phi \in \mathscr{S}(\mathbb{R}) \sim \Omega_{1}$ the solutions are piecewise smooth. The authors also give an explicit conditions on $\Omega_{1}$ :

$$
\Omega_{1}=\left\{\phi \in \mathbb{R} \mid(a(\phi(x)))^{\prime}<0,(a(\phi(x)))^{\prime \prime}=0,(a(\phi(x)))^{\prime \prime \prime}=0, x \in \mathbb{R}\right\} .
$$

Dafermos [2] introduced the concept of generalized characteristic to study the solution structures of hyperbolic conservation laws. He proved that generically the solutions generated by initial data in $C^{k}$ are piecewise smooth and do not contain centered compression waves. In other words, there is a set $\Omega_{2} \subset C^{k}$ of the first category such that for $\phi \in C^{k} \sim \Omega_{2}$ the solutions are piecewise smooth, where

$$
\Omega_{2}=\left\{\phi \in C^{k} \mid(a(\phi(x)))^{\prime}<0,(a(\phi(x)))^{\prime \prime}=0, \cdots,(a(\phi(x)))^{(k)}=0, x \in \mathbb{R}\right\} .
$$

In this paper, we first generalize Lemma 1.4 in Schaeffer [9], i.e., the case that $\left(x_{0}, t_{0}\right) \in \Gamma_{0}^{(f)}$ to the case that $\left(x_{0}, t_{0}\right) \in \bar{\Gamma}_{0}^{(f)}$. We will show that $\bar{\Gamma}_{1}$ is a union of $C^{k+1}$ curves, except the points at which at least one connected component of $\mathscr{M}$ is not an isolated point, across which the minimizing function has a jump discontinuity, $\bar{\Gamma}_{0}^{(f)}$ consists of isolated points at which the curves in $\Gamma_{1}$ begin. $\bar{\Gamma}_{0}^{(c)}$ consists of isolated points at which the curves in $\Gamma_{1}$ collide. As a result, we will prove that there is some neighborhood of $\left(x_{0}, t_{0}\right)$ such that a unique $C^{k+1}$ smooth shock originating at $\left(x_{0}, t_{0}\right)$, while there may be infinite number of intervals on which $(a(\phi(x)))^{\prime}<0,(a(\phi(x)))^{\prime \prime}=0$ (i.e., $a(\phi(x))$ is linearly decreasing) the characteristics from each of these intervals will meet at a point in any small neighborhood of $\left(x_{0}, t_{0}\right)$. We also show that for any point $\left(x_{0}, t_{0}\right) \in \bar{\Gamma}_{0}^{(c)}$ i.e. there exist finitely many, say $n$, connected components of $\mathscr{M}_{\left(x_{0}, t_{0}\right)}$, there exists a neighborhood $\Theta$ of $\left(x_{0}, t_{0}\right)$ such that $\Gamma_{1} \cap \Theta$ is the union of $n$ half shocks, $n-1$ terminating and one originating at $\left(x_{0}, t_{0}\right)$. The shocks are piecewise $C^{k+1}$ smooth and shocks are not differentiable only at points of intersection with other shocks and points which are centers of centered compression waves. The minimizing function is $C^{k}$ smooth on each $n$ components of $\Theta^{\prime} \sim \Gamma_{1}$. We prove that there is a set $\Omega_{3} \subset C^{k}(\mathbb{R})$ of the first category such that for $\phi \in C^{k}(\mathbb{R}) \sim \Omega_{3}, H=U \cup \bar{\Gamma}_{1} \cup \bar{\Gamma}_{0}^{(f)} \cup \bar{\Gamma}_{0}^{(c)}$ and the minimizing process leads to piecewise smooth solution of initial value problem (1.1) pointwise in $H$, where

$$
\begin{aligned}
\Omega_{3}= & \left\{\phi \in C^{k} \mid(a(\phi(x)))^{\prime}<0,\left(a(\phi(x))^{\prime \prime}=0, \cdots,(a(\phi(x)))^{(k)}=0,(k \geq 3) \&\right.\right. \\
& \text { there exists a point } \xi \in(x, x+\delta) \text { such that }(a(\phi(\xi)))^{\prime \prime}<0 \text { or } \\
& \text { a point } \left.\eta \in(x-\delta, x) \text { such that }(a(\phi(\eta)))^{\prime \prime}>0 \text { for each } \delta>0, x \in \mathbb{R}\right\} .
\end{aligned}
$$

Here $\Omega_{3}$ is a proper subset of $\Omega_{2}$. To our knowledge, $\Omega_{3}$ has been the smallest in the sense of inclusion relation of sets. We also prove that there is a set $\Omega_{4} \subset \mathscr{S}(\mathbb{R})$ of the first category such that for $\phi \in \mathscr{S}(\mathbb{R}) \sim \Omega_{4}$, there are only finite number of shocks. It is pointed out that piecewise smooth solutions with the underlying initial conditions may contain centered compression waves. This indicates that the class of piecewise smooth solutions with $C^{k}$ initial data excluding $\Omega_{3}$ obtained in this paper is wider than the class of the piecewise solutions with $C^{k}$ initial data excluding $\Omega_{2}$ considered by Dafermos. 
This paper is arranged as follows: we study the local structure of the solutions in Section 2 and we prove the solutions are piecewise smooth based on the results in Section 2 in Section 3.

\section{Local solution structure}

In this section, we will study the local structure of the solutions of (1.1). Some main results in this section are listed below:

- In Theorem 2.1, we study the structure of the solutions in the neighborhoods of the points at which a shock generates. We show that there is a neighborhood such that a unique $C^{k+1}$ smooth shock originates, even there are infinite number of intervals, on which $(a(\phi(x)))^{\prime}<0$, $(a(\phi(x)))^{\prime \prime}=0$, i.e., $a(\phi(x))$ is linearly decreasing, the characteristics from each of these intervals will meet at a point in any small neighborhood of $\left(x_{0}, t_{0}\right)$, while all of these points do not generate shocks.

- In Theorem 2.2, we study the points at which finitely many shocks collide to form a new shock. All of the shocks are $C^{k+1}$ smooth except at points of interaction with other shocks and points belonging to $\bar{\Gamma}_{1} \sim \Gamma_{1}$, and the shocks are not differentiable at points of interaction with other shocks and points which are centers of centered compression waves. In other words, all the shocks are piecewise $C^{k+1}$ curves.

Let $y(x, t, u)=x-t a(u)$. For each $(x, t, u) \in \mathbb{R} \times \mathbb{R}^{+} \times \mathbb{R}$, it is easy to see that $y(x, t, u)$ is just the intersection point of a straight line passing through $(x, t)$ with slope $a(u)$ with the line $t=0$. Note that

$$
F_{u}(x, t, u)=t a^{\prime}(u)\{u-\phi(x-a(u) t)\} .
$$

If $F_{u}(x, t, u)=0$, then

$$
F_{u u}(x, t, u)=t a^{\prime}(u)\left[1+(a(\phi(y(x, t, u))))^{\prime} t\right] .
$$

If $F_{u}(x, t, u)=0$ and $F_{u u}(x, t, u)=0$, then

$$
\begin{aligned}
F_{\text {uuu }}(x, t, u) & =t a^{\prime}(u)\left[(a(\phi(y(x, t, u))))^{\prime} t\right]_{u}=t^{2} a^{\prime}(u)\left[(a(\phi(y(x, t, u))))^{\prime}\right]_{u} \\
= & t^{2} a^{\prime}(u)\left[(a(\phi(y(x, t, u))))^{\prime}\right]_{y(x, t, u)}(y(x, t, u))_{u} \\
= & (-t)^{3}\left[a^{\prime}(u)\right]^{2}(a(\phi(y(x, t, u))))^{\prime \prime}
\end{aligned}
$$

By induction, if $F_{u}^{(m)}(x, t, u)=0,(m=1, \cdots, n-1)$, then we have

$$
F_{u}^{(n)}(x, t, u)=(-t)^{n}\left[a^{\prime}(u)\right]^{n-1}(a(\phi(y(x, t, u))))^{(n-1)}, \quad(n \geq 3) .
$$

In view of (2.1)-(2.4), it can be verified that if $(x, t, u)$ satisfies

$$
F_{u}^{(m)}(x, t, u)=0(m=1, \cdots, 2 n-1), \quad F_{u}^{(2 n)}(x, t, u)>0,(n \geq 2),
$$

then we have, for $y(x, t, u)=x-t a(u)$,

$$
\left\{\begin{array}{l}
(a(\phi(y(x, t, u))))^{\prime}<0, \quad(a(\phi(y(x, t, u))))^{(m)}=0, \quad(m=2,3, \cdots, 2 n-2) \\
(a(\phi(y(x, t, u))))^{(2 n-1)}>0, \quad(n \geq 2) .
\end{array}\right.
$$


On the other hand, if $x_{0}$ satisfies (2.6) then for $u=\phi\left(x_{0}\right)$,

$$
t=-\frac{1}{\left(a\left(\phi\left(x_{0}\right)\right)\right)^{\prime}}, \quad x=x_{0}-\frac{a\left(\phi\left(x_{0}\right)\right)}{\left(a\left(\phi\left(x_{0}\right)\right)\right)^{\prime}} .
$$

Consequently, (2.5) is satisfied.

\subsection{Some useful lemmas}

Lemma 2.1 ([9]) Let the set $U$ be set defined in the last section and let $u(x, t)$ be the minimizing function of $F$ defined by (1.2). Then we have

- $U$ is an open subset of $H$, and $u(x, t)$ is smooth on $U$.

- Any point $\left(x_{0}, t_{0}\right) \in \Gamma_{1}$ has a neighborhood $\Theta$ such that $\Gamma_{1} \cap \Theta$ is a smooth curve $x=\gamma(t)$ passing through $\left(x_{0}, t_{0}\right)$. The minimizing function $u(x, t)$ is smooth on both components of $\Theta \sim \Gamma_{1}$.

The above lemma is from Lemmas 1.1 and 1.2 of [9]. Let $u_{1}(x, t)$ and $u_{2}(x, t)$ be the corresponding solutions of (1.3) defined in some neighborhood $\Theta$ of $\left(x_{0}, t_{0}\right)$. We see that for nearby $(x, t)$ the minimum of $F(x, t, \bullet)$ is assumed at either $u_{1}(x, t)$ or $u_{2}(x, t)$, or both. Hence every point of $\Theta$ belongs to $U$ or $\Gamma_{1}$. Every point in the two components $\Theta_{1}$ and $\Theta_{2}$ of $\Theta \sim \Gamma_{1}$ belongs to $U$. Let $u_{i}(x, t)$ be the unique minimizing value for $F(x, t, \bullet)$ for $(x, t) \in \Theta_{i}(i=1,2)$. Any curve in $\Gamma_{1}$ which separates two components of $U$ is defined by an equation in the following form

$$
F\left(x, t, u_{2}(x, t)\right)-F\left(x, t, u_{1}(x, t)\right)=0
$$

and it follows from (1.5),(1.6) that the jump relation

$$
\dot{\gamma}(t)=[f(u)] /[u]
$$

where $[u]=u_{1}-u_{2},[f(u)]=f\left(u_{1}\right)-f\left(u_{2}\right)$ is satisfied along the curve. Thus $\gamma$ is $C^{k+1}$.

Now we turn to discuss the connection between the critical point and the characteristic. Suppose $u_{0}$ is a critical point of $F\left(x_{0}, t_{0}, \bullet\right)$, i.e. $F_{u}\left(x_{0}, t_{0}, u_{0}\right)=0$, then $u_{0}=\phi\left(x_{0}-t_{0} a\left(u_{0}\right)\right)=$ $\phi\left(y\left(x_{0}, t_{0}, u_{0}\right)\right)$ due to $(2.1)$ and so there exists a characteristic

$$
C_{0}: \quad x=y\left(x_{0}, t_{0}, u_{0}\right)+t a\left(\phi\left(y\left(x_{0}, t_{0}, u_{0}\right)\right)\right)=x_{0}+\left(t-t_{0}\right) a\left(u_{0}\right)(t>0),
$$

passing through $\left(x_{0}, t_{0}\right)$ and $F_{u}\left(x, t, u_{0}\right)=0$ for each $(x, t) \in C_{0}$. On the other hand, consider a characteristic $C_{1}: x=\bar{x}+t a(\phi(\bar{x})), t>0$ then $F_{u}(x, t, \phi(\bar{x}))=0$, for $(x, t) \in C_{1}$ due to the fact that $\phi(\bar{x})=\phi(x-t a(\phi(\bar{x})))$ and $(2.1)$. This implies that $\phi(\bar{x})$ is a critical point of $F(x, t, \bullet)$.

Naturally, it may be asked if $\phi(\bar{x})$ is a minimizing value for $F(x, t, \bullet)$ for $(x, t) \in C_{1}$. The following lemma gives an answer.

Lemma 2.2 Assume $\phi(x)$ is bounded and $C^{k}$ smooth and let

$$
C=\left\{(x, t): x=x_{0}+t a\left(\phi\left(x_{0}\right)\right), t>0\right\} .
$$

Then precisely one of the following statements must hold: 
- $C \subset U$ and $\phi\left(x_{0}\right)$ is the unique minimizing value for $F(x, t, \bullet)$; or

- there exists a point $\left(x_{1}, t_{1}\right) \in C$ such that $\phi\left(x_{0}\right)$ is either the unique minimizing value for $F\left(x_{1}, t_{1}, \bullet\right)$ which is degenerate or one of at least two minimizing values for $F\left(x_{1}, t_{1}, \bullet\right)$. Thus, $\phi\left(x_{0}\right)$ is the minimizing value for $F(x, t, \bullet)$ for $(x, t) \in C^{-}:=C \cap\left\{(x, t): t_{1}>\right.$ $t>0\} \subset U$ while $\phi\left(x_{0}\right)$ is no longer the minimizing value for $F(x, t, \bullet)$ for $(x, t) \in C^{+}:=$ $C \cap\left\{(x, t): t>t_{1}\right\}$.

Proof: First we claim that $\phi\left(x_{0}\right)$ will be no longer a minimizing value for $F(x, t, \bullet)$ for $(x, t) \in C^{+}$ if there exist at least two minimizing values for $F\left(x_{1}, t_{1}, \bullet\right)$ and $\phi\left(x_{0}\right)$ is one of them. Let $\phi\left(\tilde{x_{0}}\right) \neq \phi\left(x_{0}\right)$ be another minimizing value for $F\left(x_{1}, t_{1}, \bullet\right)$. Computing the directional derivative along $x=x_{0}+a\left(\phi\left(x_{0}\right)\right) t$ :

$$
\begin{aligned}
& \frac{d}{d \lambda}=\frac{\partial}{\partial t}+\frac{x_{1}-x_{0}}{t_{1}} \frac{\partial}{\partial x} \\
& \left.\frac{d}{d \lambda} F\left(x, t, \phi\left(x_{0}\right)\right)\right|_{\left(x_{1}, t_{1}\right)}=g\left(\phi\left(x_{0}\right)\right)=h\left(\frac{x_{1}-x_{0}}{t_{1}}\right),
\end{aligned}
$$

where

$$
h(u)=u a^{-1}(u)-f\left(a^{-1}(u)\right)=g\left(a^{-1}(u)\right),
$$

$a^{-1}(u)$ is the inverse function of $a(u)$. It can be verified that $h(u)$ is convex since $h^{\prime \prime}(u)=$ $1 / a^{\prime}(u)>0$. In fact, it follows from $x_{1}-x_{0} / t_{1}=a\left(\phi\left(x_{0}\right)\right)$ that $\phi\left(x_{0}\right)=a^{-1}\left(\left(x_{1}-x_{0}\right) / t_{1}\right)$. This gives $g\left(\phi\left(x_{0}\right)\right)=h\left(\frac{x_{1}-x_{0}}{t_{1}}\right)$. Hence

$$
\begin{aligned}
& \frac{d}{d \lambda} F\left(x, t, \phi\left(\tilde{x_{0}}\right)\right) \\
= & g\left(\phi\left(\tilde{x_{0}}\right)\right)-a\left(\phi\left(\tilde{x_{0}}\right)\right) \phi\left(x_{1}-t_{1} a\left(\phi\left(\tilde{x_{0}}\right)\right)\right)+\frac{x_{1}-x_{0}}{t_{1}} \phi\left(x_{1}-t_{1} a\left(\phi\left(\tilde{x_{0}}\right)\right)\right) \\
= & h\left(\frac{x_{1}-\tilde{x_{0}}}{t_{1}}\right)+\frac{\tilde{x_{0}}-\tilde{x_{0}}}{t_{1}} h^{\prime}\left(\frac{x_{1}-\tilde{x_{0}}}{t_{1}}\right) .
\end{aligned}
$$

Using the convexity of $h$, we get

$$
\begin{aligned}
& \left.\frac{d}{d \lambda}\left(F\left(x, t, \phi\left(x_{0}\right)\right)-F\left(x, t, \phi\left(\tilde{x_{0}}\right)\right)\right)\right|_{\left(x_{1}, t_{1}\right)} \\
= & \frac{1}{2 !} h^{\prime \prime}\left(\frac{x_{1}-\theta x_{0}-(1-\theta) \tilde{x_{0}}}{t_{1}}\right)\left(\frac{\tilde{x_{0}}-x_{0}}{t_{1}}\right)^{2}>0,
\end{aligned}
$$

where $0<\theta<1$. Thus $\phi\left(x_{0}\right)$ is no longer a minimizing value for $F(x, t, \bullet)$ for $(x, t) \in C^{+}$.

If $\phi\left(x_{0}\right)$ is a degenerate minimizing value for $F\left(x_{1}, t_{1}, \bullet\right)$, namely

$$
F_{u u}\left(x_{1}, t_{1}, \phi\left(x_{0}\right)\right)=t_{1}\left(a\left(\phi\left(x_{0}\right)\right)\right)^{\prime}\left[1+t_{1}\left(a\left(\phi\left(x_{0}\right)\right)\right)^{\prime}\right]=0 .
$$

Consequently, $F_{u}\left(x, t, \phi\left(x_{0}\right)\right)=0$ and $F_{u u}\left(x, t, \phi\left(x_{0}\right)\right)<0$ for $(x, t) \in C^{+}$, which implies that $\phi\left(x_{0}\right)$ is a local maximizing value for $F(x, t, u)$ for $(x, t) \in C^{+}$. Obviously, $\phi\left(x_{0}\right)$ is not a minimizing value for $F(x, t, u)$. By noting that $(x, t) \in C$ ( $t$ is sufficiently small) $F_{u}(x, t, u)=0$ has a unique minimizing value $\phi\left(x_{0}\right)$, and $F_{u u}\left(x, t, \phi\left(x_{0}\right)\right)>0$, the proof is completed.

Lemma 2.2 is Lemma 6 in Li-Wang [6]. For self-contained and convenience, its proof will be given below. 
Definition 2.1 A characteristic $C_{t_{c}}: x=x_{0}+a\left(\phi\left(x_{0}\right)\right) t$ starting from point $\left(x_{0}, 0\right), 0<t<$ $t_{c}(\leq \infty)$ is called valid if $\phi\left(x_{0}\right)$ is a unique minimizing value for $F(x, t, \bullet)$ for $(x, t) \in C_{t_{c}}$.

Suppose $u_{0}$ is a minimizing value for $F\left(x_{0}, t_{0}, \bullet\right)$ (not necessarily unique). Consider a characteristic $C: x=y\left(x_{0}, t_{0}, u_{0}\right)+t a\left(\phi\left(y\left(x_{0}, t_{0}, u_{0}\right)\right)\right)=x_{0}+\left(t-t_{0}\right) a\left(u_{0}\right)(t \geq 0)$, which passes through $\left(x_{0}, t_{0}\right)$ and $\left(y\left(x_{0}, t_{0}, u_{0}\right), 0\right)$. Consider the segment $C_{t_{0}}: x=x_{0}+\left(t-t_{0}\right) a\left(u_{0}\right)\left(0<t<t_{0}\right)$. It is valid according to Lemma 2.2. Therefore, a minimizing value for $F\left(x_{0}, t_{0}, \bullet\right)$ defines a part of valid characteristic passing through $\left(x_{0}, t_{0}\right)$, on the other hand a valid characteristic provides a minimizing value for $F(x, t, \bullet)$ for each given point $(x, t)$ belonging to the valid part of the characteristic. Such kind of characteristic will be often used hereafter.

Lemma 2.3 Suppose that $L_{3}=\emptyset$. Then for each point $(x, t) \in H$, there are finitely many connected components of $\mathscr{M}_{(x, t)}$.

Proof: We see each connected component of $\mathscr{M}$ is either an isolated point or a closed interval. Let $\mathscr{M}_{1}=\left[u_{i}^{-}, u_{i}^{+}\right], \mathscr{M}_{2}=\left[u_{i+1}^{-}, u_{i+1}^{+}\right]\left(u_{i}^{+}<u_{i+1}^{-}\right)$be two neighboring connected components of $\mathscr{M}$. Thus there exists a point $u_{i} \in\left(u_{i+1}^{-}, u_{i}^{+}\right)$such that

$$
F_{u}\left(x, t, u_{i}\right)=0, \quad F_{u u}\left(x, t, u_{i}\right) \leq 0,
$$

where $u_{i}$ is a local maximizing value for $F(x, t, \bullet)$. Set $y_{i}^{ \pm}=y\left(x, t, u_{i}^{ \pm}\right), y_{i+1}^{ \pm}=y\left(x, t, u_{i+1}^{ \pm}\right)$and $y_{i}=y\left(x, t, u_{i}\right)$. Now we claim

there exists a point $x_{i}^{\prime} \in\left[y_{i+1}^{-}, y_{i}\right]$ such that $\left(a\left(\phi\left(x_{i}^{\prime}\right)\right)\right)^{\prime \prime}<0$.

Otherwise, $(a(\phi(y)))^{\prime \prime} \geq 0$ for each $y \in\left[y_{i+1}^{-}, y_{i}\right]$.

Two cases need to be considered.

Case 1: $F_{u u}\left(x, t, u_{i+1}^{-}\right) \neq 0$. If this is the case, on one hand we have $\left(a\left(\phi\left(y_{i+1}^{-}\right)\right)\right)^{\prime}>-1 / t \geq$ $\left(a\left(\phi\left(y_{i}\right)\right)\right)^{\prime}$ in view of (2.10); and on the other hand $\left(a\left(\phi\left(y_{i+1}^{-}\right)\right)\right)^{\prime} \leq\left(a\left(\phi\left(y_{i}\right)\right)\right)^{\prime}$ due to (2.12). This gives a contradiction; hence Case 1 does not hold.

Case 2: $F_{u u}\left(x, t, u_{i+1}^{-}\right)=0$. In this case, since $L_{3}=\emptyset$, we have

There exists a point $\delta_{0}>0$ such that $(a(\phi(y)))^{\prime \prime} \geq 0$ for each $y \in\left[y_{i+1}^{-}, y_{i+1}^{-}+\delta_{0}\right]$ and $(a(\phi(z)))^{\prime \prime} \leq 0$ for each $z \in\left[y_{i+1}^{+}-\delta_{0}, y_{i+1}^{+}\right]$.

Next we claim that

$$
\text { there exists a point } \xi \in\left(y_{i+1}^{-}, y_{i+1}^{-}+\delta\right) \text { such that }(a(\phi(\xi)))^{\prime \prime}>0 \text { for each } \delta>0 .
$$

Otherwise according to $(2.13)$, there exists a constant $\delta_{1}>0$ such that $(a(\phi(\xi)))^{\prime \prime} \equiv 0$ for each $\xi \in\left[y_{i+1}^{-}, y_{i+1}^{-}+\delta_{1}\right]$. Set $y\left(x, t, \alpha^{\prime}\right)=y_{i+1}^{-}+\delta_{1}$, where $\alpha^{\prime}<u_{i+1}^{-}$. Then $(a(\phi(\xi)))^{\prime}=-1 / t$ for each $\xi \in\left[y_{i+1}^{-}, y\left(x, t, \alpha^{\prime}\right)\right]$, which implies $a(\phi(\xi))=-\xi / t+c$, where $c$ is a constant. Let $\xi=y_{i+1}^{-}$. We have

$$
c=\frac{1}{t}\left[y_{i+1}^{-}+t a\left(\phi\left(y_{i+1}^{-}\right)\right)\right]=\frac{x}{t},
$$

and so

$$
a(\phi(\xi))=-\frac{1}{t} \xi+\frac{x}{t}
$$


Consequently, $a(u)=a(\phi(y(x, t, u)))$, if we set $\xi=y(x, t, u)$ in $(2.15)$ and notice $y(x, t, u)=x-$ $t a(u)$ for $u \in\left[\alpha^{\prime}, u_{i+1}^{-}\right]$, which implies that $F_{u}(x, t, u) \equiv 0, F(x, t, u)$ is a constant for $u \in\left[\alpha^{\prime}, u_{i+1}^{-}\right]$. This leads to a contradiction since $\left[u_{i+1}^{-}, u_{i+1}^{+}\right]$is a connected component, hence Case 2 does not hold. Thus we complete the proof of (2.14). Similarly, we have the following conclusion

$$
\text { there exists a point } \eta \in\left(y_{i+1}^{+}-\delta, y_{i+1}^{+}\right] \text {such that }(a(\phi(\eta)))^{\prime \prime}<0 \text { for each } \delta>0 \text {. }
$$

We see $\left(a\left(\phi\left(y_{i}\right)\right)\right)^{\prime} \leq\left(a\left(\phi\left(y_{i+1}^{-}\right)\right)\right)^{\prime}=-1 / t$ since $F_{u u}\left(x, t, u_{i+1}^{-}\right)=0$. On the other hand, $\left(a\left(\phi\left(y_{i}\right)\right)\right)^{\prime}>\left(a\left(\phi\left(y_{i+1}^{-}\right)\right)\right)^{\prime}$ according to $(2.12)$ and (2.14). This is a contradiction since $\left(a\left(\phi\left(y_{i}\right)\right)\right)^{\prime} \leq$ $\left(a\left(\phi\left(y_{i+1}^{-}\right)\right)\right)^{\prime}$. Thus the assertion (2.11) is true. By a similar argument, there exists a point $x_{i}^{\prime \prime} \in\left[y_{i}, y_{i}^{+}\right]$such that $\left(a\left(\phi\left(x_{i}^{\prime \prime}\right)\right)\right)^{\prime \prime}>0$. The above arguments yield

$$
\begin{aligned}
& \text { there exists a point } x_{i}^{\prime} \in\left[y_{i+1}^{-}, y_{i}\right] \text { and a point } x_{i}^{\prime \prime} \in\left[y_{i}, y_{i}^{+}\right] \\
& \text {such that }\left(a\left(\phi\left(x_{i}^{\prime}\right)\right)\right)^{\prime \prime}<0 \text { and }\left(a\left(\phi\left(x_{i}^{\prime \prime}\right)\right)\right)^{\prime \prime}>0 .
\end{aligned}
$$

Next we claim there are only finitely many connected components of $\mathscr{M}$. If this is not true, then there exists a monotone increasing sequence $\left\{\mathscr{M}_{i}\right\}=\left(\left[u_{i}^{-}, u_{i}^{+}\right]\right)$. The sequence $\left\{\left[y_{i}^{+}, y_{i}^{-}\right]\right\}$is monotone decreasing and bounded, then it must be convergent to a point $x_{0}$. It is easy to know that

$$
\lim _{i \rightarrow \infty} x_{i}^{\prime}=\lim _{i \rightarrow \infty} x_{i}^{\prime \prime}=\lim _{i \rightarrow \infty} y_{i}=x_{0} .
$$

It follows from $\left(a\left(\phi\left(y_{i}\right)\right)\right)^{\prime} \leq-1 / t$ that $\left(a\left(\phi\left(x_{0}\right)\right)\right)^{\prime} \leq-\frac{1}{t}<0$. On the other hand, $\left(a\left(\phi\left(x_{0}\right)\right)\right)^{\prime \prime}=$ $0, \cdots,\left(a\left(\phi\left(x_{0}\right)\right)\right)^{(k)}=0$ in light of $(2.17)$. For each $\delta>0$, there exist $i_{0}>0$ such that $\left[y_{i}^{+}, y_{i}^{-}\right] \subset$ $\left(x_{0}-\delta, x_{0}+\delta\right)$ for each $i>i_{0}$. We see $x_{0}<x_{i}^{\prime}<x_{i}^{\prime \prime}$ for each $i>i_{0}$ since the sequence $\left\{\left[y_{i}^{+}, y_{i}^{-}\right]\right\}$ is monotone decreasing. Observing $\left(a\left(\phi\left(x_{i}^{\prime}\right)\right)\right)^{\prime \prime}<0, x_{i}^{\prime} \in\left(x_{0}, x_{0}+\delta\right)$, which implies $x_{0} \in L_{3}$. This is contradicts $L_{3}=\emptyset$. The proof is completed.

Same result by Li and Wang [7] to Lemma 2.3 has been obtained under the hypothesis that $\phi$ is locally finite to $\mathrm{f}$.

Below we give some lemmas which will be used in the proof of one of our main results, Theorem 2.1 .

Lemma 2.4 The locus of the degenerate points on all the characteristics originating from some neighborhood of $\left[y\left(x_{0}, t_{0}, \beta\right), y\left(x_{0}, t_{0}, \alpha\right)\right]$ form two half curves in the neighborhood of $\left(x_{0}, t_{0}\right)$, for $t>t_{0}$ with a unique common point $\left(x_{0}, t_{0}\right)$. Each of them is continuous and consists of union of countable open arcs, on which the curve is $C^{k}$ and points as the end point of the arcs on which the curve is not differentiable but the left derivative continuous on the left and right derivative continuous on the right. The graphs of them are demonstrated in Figure 1.

Proof: Note that by $(2.1)$ the critical set of $F(x, t, \bullet)$ is contained in the compact interval $J=\{u:|u| \leq M\}$, where $M=\sup _{y}|\phi(y)|$. First we claim that

for each open interval $(\alpha-\varepsilon, \beta+\varepsilon)$, there exists an open neighborhood $\Theta$ of $\left(x_{0}, t_{0}\right)$ such that all the minimizing values for $F(x, t, \bullet)$ belong to $(\alpha-\varepsilon, \beta+\varepsilon)$ for $(x, t)$ belonging to $\Theta$.

Otherwise there exists a sequence $\left(x_{n}, t_{n}\right)$ convergent to $\left(x_{0}, t_{0}\right)$ and a sequence $u_{n}$ belonging to $J \sim(\alpha-\varepsilon, \beta+\varepsilon)$ such that $F\left(x_{n}, t_{n}, u_{n}\right)=\min _{u \in \mathbb{R}} F\left(x_{n}, t_{n}, u\right)(n=1,2, \ldots)$. Since the set 


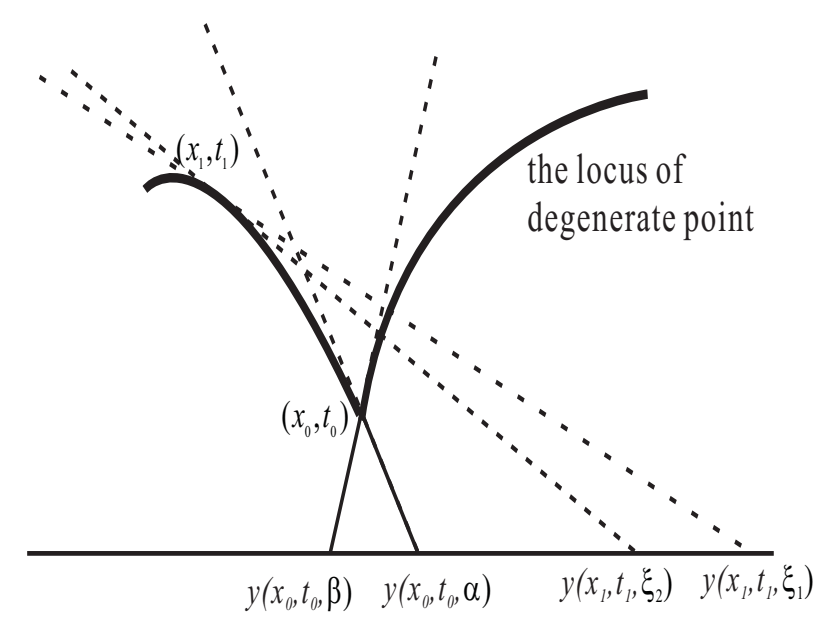

Figure 1: The locus of the degenerate points on all the characteristics. The interval $\left(y\left(x_{1}, t_{1}, \xi_{2}\right), y\left(x_{1}, t_{1}, \xi_{1}\right)\right)$ is the linearly decreasing interval, on which $(a(\phi(\bullet)))^{\prime \prime}=0$ and $(a(\phi(\bullet)))^{\prime}<0$. There may be infinitely many such intervals in the neighborhood of $\left(y\left(x_{1}, t_{1}, \xi_{2}\right), y\left(x_{1}, t_{1}, \xi_{1}\right)\right)$.

$J \sim(\alpha-\varepsilon, \beta+\varepsilon)$ is compact and we can select a subsequence of $u_{n}$ written again as $u_{n}$ for convenience convergent to $u_{1}$ belonging to the set $J \sim(\alpha-\varepsilon, \beta+\varepsilon)$. Then $F\left(x_{0}, t_{0}, u_{0}\right)=$ $\lim _{n \rightarrow \infty} F\left(x_{n}, t_{n}, u_{n}\right)=F\left(x_{0}, t_{0}, u_{1}\right)$, since the function $m(x, t)=\min _{u \in \mathbb{R}} F(x, t, u)$ is continuous of $x$ and $t$ (see Lemma A.1 in the Appendix). This implies that there are at least two connected components of $\mathscr{M}_{\left(x_{0}, t_{0}\right)}$. It is a contradiction since there is a unique connected component of $\mathscr{M}_{\left(x_{0}, t_{0}\right)}$. Thus the assertion (2.18) is true. According to (2.18), we can find a sufficiently small constant $\varepsilon_{0}>0$ and a neighborhood $\Theta$ of $\left(x_{0}, t_{0}\right)$ such that for each $(x, t) \in \Theta, u \in\left(\alpha-\varepsilon_{0}, \beta+\varepsilon_{0}\right)$, $x-t a(u)$ belongs to $\left(y\left(x_{0}, t_{0}, \beta\right)-\delta_{0}, y\left(x_{0}, t_{0}, \alpha\right)+\delta_{0}\right)$.

Two half curves mentioned above can be written in the following form

$$
\begin{aligned}
& \left\{\begin{array}{l}
x(\xi)=\xi-\frac{a(\phi(\xi))}{(a(\phi(\xi)))^{\prime}} \\
t(\xi)=-\frac{1}{(a(\phi(\xi)))^{\prime}}
\end{array} \quad \xi \in\left[y\left(x_{0}, t_{0}, \alpha\right), y\left(x_{0}, t_{0}, \alpha\right)+\delta_{0}\right]\right. \\
& \left\{\begin{array}{l}
x(\xi)=\xi-\frac{a(\phi(\xi))}{(a(\phi(\xi)))^{\prime}} \\
t(\xi)=-\frac{1}{(a(\phi(\xi)))^{\prime}}
\end{array} \quad \xi \in\left[y\left(x_{0}, t_{0}, \beta\right)-\delta_{0}, y\left(x_{0}, t_{0}, \beta\right)\right] .\right.
\end{aligned}
$$

We see they have a common point $\left(x_{0}, t_{0}\right)$. Moreover, the slope of the line tangent to the curve $(2.19)$ at $\left(x_{0}, t_{0}\right)$ is $a(\alpha)$ and the slope of the line tangent to the curve $(2.20)$ at $\left(x_{0}, t_{0}\right)$ is $a(\beta)$. By a direct computation and in light of (2.2), we have

$$
F_{u}(x(\xi), t(\xi), \phi(\xi))=0, \quad F_{u u}(x(\xi), t(\xi), \phi(\xi))=0 .
$$

Thus, the curve (2.19) is the locus of the degenerate points on all the characteristics that start from the interval $\left[y\left(x_{0}, t_{0}, \alpha\right), y\left(x_{0}, t_{0}, \alpha\right)+\delta_{0}\right]$ and the curve $(2.20)$ is the locus of the degenerate 
points on all the characteristics that start from the interval $\left[y\left(x_{0}, t_{0}, \beta\right)-\delta_{0}, y\left(x_{0}, t_{0}, \beta\right)\right]$. Let $\left(x_{l}, t_{1}\right),\left(x_{r}, t_{1}\right) \in \Theta\left(t_{1}>t_{0}\right)$ be the intersection points of the line $t=t_{1}$ with the curves $(2.19)$ and (2.20). Now we claim $x_{r}-x_{l}>0$. The proof can be found in Lemma A.2 of the Appendix. Thus these two curves have a unique common point $\left(x_{0}, t_{0}\right)$. Each of them is continuous and consists of union of countable open arcs, on which the curve is $C^{k}$ and points as the end point of the arcs on which the curve is not differentiable but the left derivative continuous on the left and right derivative continuous on the right; the proof this statement will be postponed to Lemma A.4 in Appendix.

Lemma 2.5 All the characteristics that originate from the interval $\left(y\left(x_{0}, t_{0}, \alpha\right), y\left(x_{0}, t_{0}, \alpha\right)+\delta_{0}\right)$ $\left(\left(y\left(x_{0}, t_{0}, \beta\right)-\delta_{0}, y\left(x_{0}, t_{0}, \beta\right)\right)\right)$ can only intersect with each other after the time $t_{0}$ and for any two characteristics that originate from the points on the right (left) of $\left(y\left(x_{0}, t_{0}, \alpha\right), 0\right)\left(\left(y\left(x_{0}, t_{0}, \beta\right), 0\right)\right)$, the time when the one closer to $\left(y\left(x_{0}, t_{0}, \alpha\right), 0\right)\left(\left(y\left(x_{0}, t_{0}, \beta\right), 0\right)\right)$ touches the curve $(2.19)((2.20))$ is not later than the time when they intersect with each other, where $\delta_{0}>0$ is a constant.

Proof: Consider the following two characteristics:

$$
\begin{aligned}
& x=\bar{x}_{1}+\operatorname{ta}\left(\phi\left(\bar{x}_{1}\right)\right), \\
& x=\bar{x}_{2}+\operatorname{ta}\left(\phi\left(\bar{x}_{2}\right)\right),
\end{aligned}
$$

where $y\left(x_{0}, t_{0}, \alpha\right)<\bar{x}_{1}<\bar{x}_{2}<y\left(x_{0}, t_{0}, \alpha\right)+\delta_{0}$. Suppose $\left(x_{12}, t_{12}\right)$ is the intersection point of them; i.e.,

$$
t_{12}=-\frac{\bar{x}_{1}-\bar{x}_{2}}{a\left(\phi\left(\bar{x}_{1}\right)\right)-a\left(\phi\left(\bar{x}_{2}\right)\right)}=-\frac{1}{\left(a\left(\phi\left(\bar{x}_{12}\right)\right)\right)^{\prime}},
$$

where $\bar{x}_{1}<\bar{x}_{12}<\bar{x}_{2}$. Let $t_{1}=-1 /\left(a\left(\phi\left(\bar{x}_{1}\right)\right)\right)^{\prime}$ and $t_{2}=-1 /\left(a\left(\phi\left(\bar{x}_{2}\right)\right)\right)^{\prime}$. Thus $F_{u}\left(x_{i}, t_{i}, \phi\left(\bar{x}_{i}\right)\right)=0$ and $F_{u u}\left(x_{i}, t_{i}, \phi\left(\bar{x}_{i}\right)\right)=0$, where $x_{i}=\bar{x}_{i}+t_{i} a\left(\phi\left(\bar{x}_{i}\right)\right)(i=1,2)$. It can be verified that

$$
t_{0}<t_{1} \leq t_{12} \leq t_{2}
$$

according to (2.13) and (2.14). A similar conclusion can also be obtained if $\bar{x}_{1}, \bar{x}_{2} \in\left(y\left(x_{0}, t_{0}, \beta\right)-\right.$ $\left.\delta_{0}, y\left(x_{0}, t_{0}, \beta\right)\right)$. This implies that all the characteristics that originate from the intervals $\left(y\left(x_{0}, t_{0}, \alpha\right)\right.$, $\left.y\left(x_{0}, t_{0}, \alpha\right)+\delta_{0}\right)$ and $\left(\left(y\left(x_{0}, t_{0}, \beta\right)-\delta_{0}, y\left(x_{0}, t_{0}, \beta\right)\right)\right)$ can only intersect with each other after $t=t_{0}$; and for any two characteristics that originate from the points on the right (left) of $\left(y\left(x_{0}, t_{0}, \alpha\right), 0\right)$ and $\left(\left(y\left(x_{0}, t_{0}, \beta\right), 0\right)\right)$, the time when the one closer to $\left(y\left(x_{0}, t_{0}, \alpha\right), 0\right)$ and $\left(\left(y\left(x_{0}, t_{0}, \beta\right), 0\right)\right)$ touches the curve (2.19) ((2.20)) is not later than the time when they intersect with each other. Thus we complete the proof.

Lemma 2.6 All the minimizing values for $F(x, t, \bullet)$ are non-degenerate for $(x, t) \in \Theta^{\prime}$ and there exists a unique minimizing value for $F(x, t, \bullet)$ for $(x, t) \in \Theta^{\prime}$ lying under and on the curves (2.19) and (2.20).

Proof: We consider two possible cases.

Case 1: under the curves. There is a unique minimizing value for $F(x, t, \bullet)$ for $(x, t) \in$ $\Theta^{\prime} \cap\left\{t \leq t_{0}\right\}$ which is non-degenerate due to Lemma 2.5. Consider a point $\left(x_{1}, t_{1}\right) \in \Theta$, if $x_{1}<x_{l}\left(x_{1}>x_{r}\right)$. Then if follows from Lemma 2.5 that there exists a unique minimizing value 
for $F\left(x_{1}, t_{1}, \bullet\right)$ which is non-degenerate.

Case 2: on the curves. If $x_{1}=x_{l}\left(x_{1}=x_{r}\right)$, we claim that

there exists a unique minimizing value for $F\left(x_{1}, t_{1}, \bullet\right)$ and it is non-degenerate.

In fact, there exists a unique minimizing value $u_{1}$ for $F\left(x_{1}, t_{1}, \bullet\right)$ such that $y\left(x_{1}, t_{1}, u_{1}\right)$ belongs to $\left(y\left(x_{0}, t_{0}, \beta\right)-\delta_{0}, y\left(x_{0}, t_{0}, \beta\right)\right)$ according to Lemma 2.5. If there exists another minimizing value $u_{2}$ for $F\left(x_{1}, t_{1}, \bullet\right)$ such that $y\left(x_{1}, t_{1}, u_{2}\right)$ belongs to $\left(y\left(x_{0}, t_{0}, \alpha\right), y\left(x_{0}, t_{0}, \alpha\right)+\delta_{0}\right)$. Then there exists a characteristic passing through $\left(x_{1}, t_{1}\right)$ :

$$
x=y\left(x_{1}, t_{1}, u_{2}\right)+t a\left(\phi\left(y\left(x_{1}, t_{1}, u_{2}\right)\right)\right), \quad 0<t \leq t_{1} .
$$

Let

$$
x=\xi+t a(\phi(\xi)), \quad 0<t \leq t_{1}
$$

be the line tangent to the curve (2.19) from below at the point $\left(x_{1}, t_{1}\right)$. Since

$$
t_{1}=-\frac{1}{(a(\phi(\xi)))^{\prime}}=-\frac{1}{\left(a\left(\phi\left(y\left(x_{1}, t_{1}, u_{2}\right)\right)\right)\right)^{\prime}}
$$

according to the argument of assertion (2.14), we know if $(a(\phi(\xi)))^{\prime}<0,(a(\phi(\xi)))^{\prime \prime}=0$, for each $\xi \in\left[\xi_{1}, \xi_{2}\right]$, then all the characteristics that start from the interval $\left[\xi_{1}, \xi_{2}\right]$ meet at a point, say $(x, t)$ and so $F(x, t, u)$ is a constant for each $u \in\left[\phi\left(\xi_{2}\right), \phi\left(\xi_{1}\right)\right]$, thus we have $F\left(x_{1}, t_{1}, \phi(\xi)\right)=$ $F\left(x_{1}, t_{1}, u_{2}\right)$, which implies $\phi(\xi)$ is a minimizing value for $F\left(x_{1}, t_{1}, \bullet\right)$. Thus the characteristic (2.26) is valid.

Let $u_{1}$ and $\left[u_{3}, \phi(\xi)\right]$ be the connected components of $\mathscr{M}_{\left(x_{1}, t_{1}\right)}$. In light of Lemma A.3 in Appendix, there exists an open neighborhood $\bar{\Theta}$ of $\left(x_{1}, t_{1}\right)$ such that $\bar{\Theta} \cap \Gamma_{1}$ contains a half curve $x=\gamma^{-}(t)$ terminating at $\left(x_{1}, t_{1}\right)$. Furthermore, we have

$$
\frac{d \gamma^{-}(t)}{d t}=\frac{f\left(u_{1}(x, t)\right)-f\left(u_{2}(x, t)\right)}{u_{1}(x, t)-u_{2}(x, t)}
$$

$y\left(x, t, u_{1}(x, t)\right) \in\left(y\left(x_{1}, t_{1}, u_{1}\right), y\left(x_{1}, t_{1}, u_{1}\right)+\delta\right), y\left(x, t, u_{2}(x, t)\right) \in(\xi-\delta, \xi),\left(t<t_{1}\right)$. Let $t \rightarrow t_{1}-$, we have

$$
\lim _{t \rightarrow t_{1}-0} \frac{d \gamma^{-}(t)}{d t}=\frac{f\left(u_{1}\right)-f(\phi(\xi))}{u_{1}-\phi(\xi)} \in\left(a(\phi(\xi)), a\left(u_{1}\right)\right) .
$$

On the other hand, the slope of the line tangent to the curve $(2.19)$ at $(x, t)$ tending to $\left(x_{1}, t_{1}\right)$ is convergent to $a(\phi(\xi))$. Consequently the curve $x=\gamma^{-}(t)$ lies between the characteristic (2.26) and the curve (2.19) for $t$ close to $t_{1}$. Consider a point $(\tilde{x}, \tilde{t})=\left(\gamma^{-}(\tilde{t}), \tilde{t}\right)$, there exists a minimizing value $u(\tilde{x}, \tilde{t})$ for $F(\tilde{x}, \tilde{t}, \bullet)$ such that $y(\tilde{x}, \tilde{t}, u(\tilde{x}, \tilde{t})) \in(\xi-\delta, \xi)$, thus there exists a valid characteristic passing through $(\tilde{x}, \tilde{t})$. On the other hand, it touches the curve (2.19) and becomes invalid according to Lemma 2.2, which is a contradiction. Hence the assertion (2.24) is true.

It is easy to see that the shock will not touch the curves (2.19) and (2.20), which implies the shock lies between these two curves, so it must be smooth. 


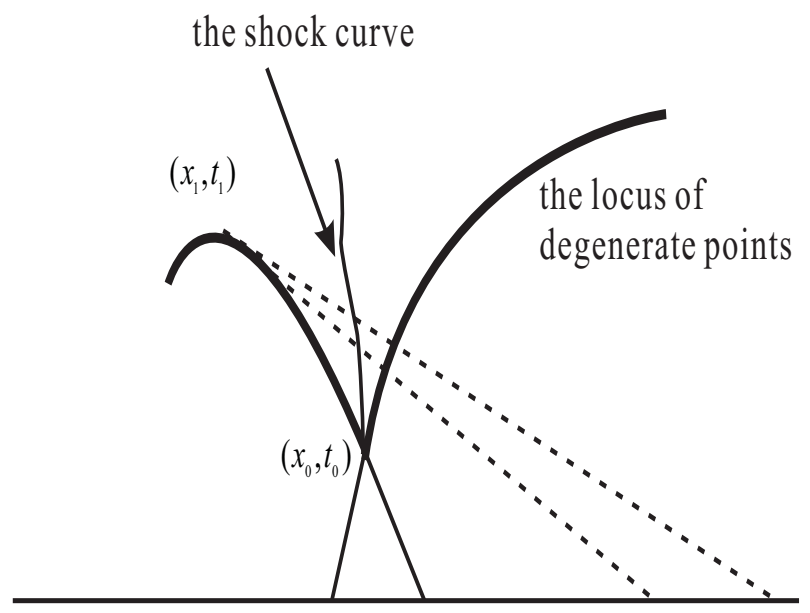

Figure 2: The local structure of the solution.

\subsection{Shock generation results}

Theorem 2.1 Assume $L_{3}=\emptyset$ and suppose that there is a unique connected component $[\alpha, \beta]$ of $\mathscr{M}_{\left(x_{0}, t_{0}\right)}$, and $F_{u u}\left(x_{0}, t_{0}, \alpha\right)=0$ then $\left(x_{0}, t_{0}\right)$ has a neighborhood $\Theta$ such that $\Gamma_{1} \cap \Theta$ consists of a half-curve originating at $\left(x_{0}, t_{0}\right)$. The minimizing function is smooth on $\Theta^{\prime} \sim \Gamma_{1}$. The local structure of the solution is given in Figure 2.

Proof. We will prove that there exists a $C^{k+1}$ smooth shock originating at $\left(x_{0}, t_{0}\right)$. For each given $t_{1}>t_{0}$, let $A_{t_{1}}=\left\{x \mid\left(x, t_{1}\right) \in \Theta\right.$, there exists a valid characteristic which originates from a point on the left of the point $\left(y\left(x_{0}, t_{0}, \beta\right), 0\right)$ and passes through $\left.\left(x, t_{1}\right)\right\} . B_{t_{1}}=\left\{x \mid\left(x, t_{1}\right) \in \Theta\right.$, there exists a valid characteristic which originates from a point on the right of the point $\left(y\left(x_{0}, t_{0}, \alpha\right), 0\right)$ and passes through $\left.\left(x, t_{1}\right)\right\}$. According to the assertion (2.24), there exists a unique valid characteristic passing through $\left(x_{l}, t_{1}\right)$, then $\left(x_{l}, t_{1}\right) \in A_{t_{1}}$, which implies that $A_{t_{1}} \neq \emptyset$. Similar, $B_{t_{1}} \neq \emptyset$. Let $x_{1}$ be the supremum of the set $A_{t_{1}}$ and $x_{2}$ be the infimum of the set $B_{t_{1}}$.

Next we will show:

There are two valid characteristics passing through $\left(x_{1}, t_{1}\right)$. One originates from a point on the left of the point $\left(y\left(x_{0}, t_{0}, \beta\right), 0\right)$ the other originates from a point on the right of the point $\left(y\left(x_{0}, t_{0}, \alpha\right), 0\right)$.

First we claim that there are at least two minimizing values for $F\left(x_{1}, t_{1}, \bullet\right)$.

To see this, if there is a unique minimizing value $u_{1}$ of $u$ for $F\left(x_{1}, t_{1}, \bullet\right)$ then there exists a unique characteristic passing through $\left(x_{1}, t_{1}\right)$, which satisfies one of the following cases:

case 1: it originates from a point on the left of the point $\left(y\left(x_{0}, t_{0}, \beta\right), 0\right)$ and $u_{1}$ is non-degenerate. In this case, according to the Lemma 2.1, we can find a neighborhood $U\left(x_{1}\right)$ of $x_{1}$ such that for each $x \in U\left(x_{1}\right),\left(x, t_{1}\right)$, there exists only one characteristic that originates from a point on the left of the point $\left(y\left(x_{0}, t_{0}, \beta\right), 0\right)$. Since $x_{1}$ is the supremum of the set $A_{t_{1}}$, there exists a point $\tilde{x} \in U\left(x_{1}\right)$ such that there exists a characteristic passing through $\left(\tilde{x}, t_{1}\right)$ that originates from a 
point on the left of the point $\left(y\left(x_{0}, t_{0}, \beta\right), 0\right)$. This is contradictory to the property of $U\left(x_{1}\right)$.

case 2: it originates from a point on the right of the point $\left(y\left(x_{0}, t_{0}, \alpha\right), 0\right)$. Similar to the case 1 above, a contradiction can also be obtained.

Next, we claim that

$$
\text { there are at most two minimizing values for } F\left(x_{1}, t_{1}, \bullet\right)
$$

Otherwise, there exist two minimizing values $u_{1}$ and $u_{2}$ for $F\left(x_{1}, t_{1}, \bullet\right)$ such that $y\left(x_{1}, t_{1}, u_{1}\right)=$ $x_{1}-t_{1} a\left(u_{1}\right)$ and $y\left(x_{1}, t_{1}, u_{2}\right)=x_{1}-t_{1} a\left(u_{2}\right)$ belong to $\left(y\left(x_{0}, t_{0}, \beta\right)-\delta_{0}, y\left(x_{0}, t_{0}, \beta\right)\right)$ or $\left(y\left(x_{0}, t_{0}, \alpha\right), y\left(x_{0}, t_{0}, \alpha\right)+\right.$ $\left.\delta_{0}\right)$. Suppose $y\left(x_{1}, t_{1}, u_{1}\right), y\left(x_{1}, t_{1}, u_{2}\right)$ belong to $\left(y\left(x_{0}, t_{0}, \alpha\right), y\left(x_{0}, t_{0}, \alpha\right)+\delta_{0}\right)$. Then there exist two characteristics

$$
\begin{aligned}
& x=\bar{x}_{1}+t a\left(\phi\left(\bar{x}_{1}\right)\right) \\
& x=\bar{x}_{2}+t a\left(\phi\left(\bar{x}_{2}\right)\right)
\end{aligned}
$$

and they are valid, where $\bar{x}_{1}<\bar{x}_{2}$. Suppose that $(\bar{x}, \bar{t})$ is the intersection point of them. Then we have

$$
\bar{x}=\frac{\bar{x}_{1} a\left(\phi\left(\bar{x}_{2}\right)\right)-\bar{x}_{2} a\left(\phi\left(\bar{x}_{1}\right)\right)}{a\left(\phi\left(\bar{x}_{2}\right)\right)-a\left(\phi\left(\bar{x}_{1}\right)\right)}, \quad \bar{t}=\frac{\bar{x}_{1}-\bar{x}_{2}}{a\left(\phi\left(\bar{x}_{2}\right)\right)-a\left(\phi\left(\bar{x}_{1}\right)\right)} .
$$

Let

$$
\overline{\bar{t}}=-\frac{1}{\left(a\left(\phi\left(\bar{x}_{1}\right)\right)\right)^{\prime}}, \quad \overline{\bar{x}}=\bar{x}_{1}-\frac{a\left(\phi\left(\bar{x}_{1}\right)\right)}{\left(a\left(\phi\left(\bar{x}_{1}\right)\right)\right)^{\prime}} .
$$

Then $(\overline{\bar{x}}, \overline{\bar{t}})$ lies on the characteristic $(2.31), \bar{t} \geq \overline{\bar{t}}$ and $(\overline{\bar{x}}, \overline{\bar{t}})$ satisfies

$$
F_{u}\left(\overline{\bar{x}}, \overline{\bar{t}}, \phi\left(x_{1}\right)\right)=0, \quad F_{u u}\left(\overline{\bar{x}}, \overline{\bar{t}}, \phi\left(x_{1}\right)\right)=0 .
$$

Hence, $\phi\left(x_{1}\right)$ is a degenerate point of $F(\overline{\bar{x}}, \overline{\bar{t}}, \bullet)$. It follows from Lemma 2.2 that $\phi\left(x_{1}\right)$ is no longer the minimum point of $F(x, t, \bullet)$ for $(x, t)$ belonging to the characteristics $x=x_{1}+\operatorname{ta}\left(\phi\left(x_{1}\right)\right)($ $t>\overline{\bar{t}}$ ). If $\bar{t}>\overline{\bar{t}}$, then the characteristic (2.31) becomes invalid when $t>\overline{\bar{t}}$. This is contradictory to the fact that the characteristic $(2.31)$ is valid. If $\bar{t}=\overline{\bar{t}}$, then $\phi\left(\overline{x_{1}}\right)$ is a degenerate minimizing value for $F\left(x_{1}, t_{1}, \bullet\right)$ which is a contradiction according to the assertion (2.24).

Combining (2.29) and (2.30), we have the following conclusion

$$
\begin{aligned}
& \text { there are only two minimizing values for } F\left(x_{1}, t_{1}, \bullet\right) \text { and they are } \\
& \text { non-degenerate, which implies there are two valid characteristics passing through }
\end{aligned}
$$
$\left(x_{1}, t_{1}\right)$. One originates from a point on the left of the point $\left(y\left(x_{0}, t_{0}, \beta\right), 0\right)$ the other originates from a point on the right of the point $\left(y\left(x_{0}, t_{0}, \alpha\right), 0\right)$.

By a similar argument to the assertion (2.28), we have

There are two valid characteristics passing through $x_{2}$. One originates from a point on the left of the point $\left(y\left(x_{0}, t_{0}, \beta\right), 0\right)$ the other originates from a point on the right of the point $\left(y\left(x_{0}, t_{0}, \alpha\right), 0\right)$.

Now we claim

$$
x_{1}=x_{2} \text {. }
$$


If not, i.e., $x_{1} \neq x_{2}$, then it follows from the assertions (2.28) and (2.36) that there is a valid characteristic passing through $\left(x_{1}, t_{1}\right)$ and a valid characteristic passing through $\left(x_{2}, t_{1}\right)$ such that they intersect with each other at a time earlier than $t_{1}$. This is a contradiction since they will become invalid after the time when they intersect according to Lemma 2.2. Therefore $x_{1}=x_{2}$. In this way a unique curve $x=\gamma(t)$ is defined for $t \geq t_{0}$. Therefor there are only two minimizing values for $F(\gamma(t), t, \bullet)$.

Consider any point $\left(x_{1}, t_{1}\right) \in \Theta \cap\left\{t>t_{0}\right\}$, if $x_{1}<\gamma\left(t_{1}\right)\left(x_{1}>\gamma\left(t_{1}\right)\right)$, We see that there is a unique value of $\mathrm{u}$ which minimizes $F\left(x_{1}, t_{1}, \bullet\right)$ since it lies on the left of the point $\left(\gamma\left(t_{1}\right), t_{1}\right)$, thus the minimizing function is smooth at the point $(x, t)$ in view of Lemma 2.1. If $x_{1}=\gamma\left(t_{1}\right)$, then there are only two values of $u$ which minimizes $F\left(x_{1}, t_{1}, \bullet\right)$ and they are non-degenerate according to (2.35). Combining Lemma 2.2 and the above arguments, we complete the proof.

Remark 2.1 Lemma 1.4 in Schaeffer [9] is a special case of Theorem 2.1, even if we set $\alpha=\beta$ in Theorem 2.1. Theorem 2.1 shows that even around a point or an interval $\left[y\left(x_{0}, t_{0}, \beta\right), y\left(x_{0}, t_{0}, \alpha\right)\right]$ on $t=0$, there can be infinite number of intervals, on which $(a(\phi(x)))^{\prime}<0,(a(\phi(x)))^{\prime \prime}=0$, i.e., $a(\phi(x))$ is linearly decreasing, the characteristics from each of these intervals will meet at a point in any small neighborhood of $\left(x_{0}, t_{0}\right)$, no shocks generate at all of these points, while there is only a $C^{k+1}$ smooth shock in this neighborhood. On the other hand, this theorem implies our solutions contain centered compression waves.

Theorem 2.2 Assume $L_{3}=\emptyset$. If $\left[u_{1}^{-}, u_{1}^{+}\right],\left[u_{2}^{-}, u_{2}^{+}\right], \cdots,\left[u_{n}^{-}, u_{n}^{+}\right]$are the $n$ connected components of $\mathscr{M}_{\left(x_{0}, t_{0}\right)}$ (suppose $\left.u_{1}^{-} \leq u_{1}^{+}<u_{2}^{-} \leq u_{2}^{+} \cdots<u_{n}^{-} \leq u_{n}^{+}\right)$, where $n \geq 2$, then $\left(x_{0}, t_{0}\right)$ has a neighborhood $\Theta$ such that $\Gamma_{1} \cap \Theta$ consists of $n$ half-curves, one originating at $\left(x_{0}, t_{0}\right)$ and the other $(n-1)$ terminating at $\left(x_{0}, t_{0}\right)$. Moreover, the minimizing function is smooth on $\Theta^{\prime} \sim \Gamma_{1}$.

Proof: Set

$$
\begin{aligned}
& l_{i}^{+}: x=y\left(x_{0}, t_{0}, u_{i}^{+}\right)+\operatorname{ta}\left(\phi\left(y\left(x_{0}, t_{0}, u_{i}^{+}\right)\right)\right) \\
& l_{i}^{-}: x=y\left(x_{0}, t_{0}, u_{i}^{-}\right)+\operatorname{ta}\left(\phi\left(y\left(x_{0}, t_{0}, u_{i}^{-}\right)\right)\right)
\end{aligned}
$$

where $i=1, \cdots, n, 0<t<t_{0}$. By an argument similar to the proof of assertion (2.18), there exists a neighborhood $\Theta$ of $\left(x_{0}, t_{0}\right)$ and $n$ intervals $\left(y\left(x_{0}, t_{0}, u_{i}^{+}\right)-\delta, y\left(x_{0}, t_{0}, u_{i}^{-}\right)+\delta\right)(i=1, \cdots, n)$, $\delta<\frac{1}{2} \min _{1 \leq i \leq n-1}\left\{a\left(u_{i+1}^{-}\right)-a\left(u_{i}^{+}\right)\right\}$such that for each given $(x, t) \in \Theta$ and each minimizing value $u(x, t)$ for $F(x, t, \bullet), y(x, t, u(x, t))$ belongs to $\left(y\left(x_{0}, t_{0}, u_{i}^{+}\right)-\delta, y\left(x_{0}, t_{0}, u_{i}^{-}\right)+\delta\right)$ for some $i \in$ $\{1, \cdots, n\}$. In light of Lemma A.3 in Appendix, we can deduce that all the minimizing values for $F(x, t, \bullet)$ are non-degenerate for $(x, t) \in \Theta^{\prime} \cap\left\{t \leq t_{0}\right\}$ here $\Theta^{\prime}=\Theta \sim\left\{\left(x_{0}, t_{0}\right)\right\}$. Furthermore, there exists a unique half-curve terminating at $\left(x_{0}, t_{0}\right)$ denoted as $x=\gamma_{i}^{-}(t)$ is defined for $t \leq t_{0}$, which lies in the triangle domain $G_{i+1}^{i}$ formed by the line $t=0$, the characteristic $l_{i}^{+}$and $l_{i+1}^{-}$, moreover there exist two minimizing values for $F(x, t, \bullet)$ and they are non-degenerate ,where $x=\gamma_{i}^{-}(t)(i=1, \cdots, n-1)$, according to Lemma A.3 in Appendix. By the same way used in Theorem 2.1, there exists a half-curve originating at $\left(x_{0}, t_{0}\right)$ denoted as $x=\gamma^{+}(t),\left(t \geq t_{0}\right)$. Thus, we complete the proof.

Corollary 2.1 Any point $\left(x_{0}, t_{0}\right) \in \bar{\Gamma}_{1}$ has a neighborhood $\Theta$ such that $\bar{\Gamma}_{1} \cap \Theta$ is a curve $x=\gamma(t)$ passing through $\left(x_{0}, t_{0}\right)$ and $x=\gamma(t)$ is $C^{k+1}$ smooth at each point except $t=t_{0}$. The minimizing function $u(x, t)$ is smooth on both components of $\Theta \sim \bar{\Gamma}_{1}$. 
The smoothness of the curve $x=\gamma(t)$ can be decided by the following cases:

Case 1: $\mathscr{M}=\left\{u_{1}, u_{2}\right\}$ for $F\left(x_{0}, t_{0}, \bullet\right)$. In this case,

- If $F_{u u}\left(x_{0}, t_{0}, u_{1}\right)=0$ or $F_{u u}\left(x_{0}, t_{0}, u_{2}\right)=0$, then the curve $x=\gamma(t)$ is $C^{1}$ smooth. In fact, $x=\gamma(t)$ is only $C^{1}$ at the point $t=t_{0}$ since $u_{1}(x, t)$ and $u_{2}(x, t)$ are continuous, but $u_{1 x}\left(x, t_{0}\right) \rightarrow \infty$ or $u_{2 x}\left(x, t_{0}\right) \rightarrow \infty$ as $x \rightarrow x_{0}-o$ or $x \rightarrow x_{0}+o$ although it is $C^{k+1}$ at each point except $t=t_{0}$.

- If $F_{u u}\left(x_{0}, t_{0}, u_{1}\right) \neq 0$ and $F_{u u}\left(x_{0}, t_{0}, u_{2}\right) \neq 0$, then the curve $x=\gamma(t)$ is $C^{k+1}$ smooth.

Case 2: $\mathscr{M}=\left[u_{1}^{-}, u_{1}^{+}\right] \cup\left[u_{2}^{-}, u_{2}^{+}\right]$, where $u_{1}^{-}<u_{1}^{+}$or $u_{2}^{-}<u_{2}^{+}$. In this case, the curve $x=\gamma(t)$ is continuous at the point $t=t_{0}$.

Throughout this section, we can see the shocks are piecewise $C^{k+1}$ smooth except at points of interaction with other shocks and points belonging to $\bar{\Gamma}_{1} \sim \Gamma_{1}$, and the shocks are not differentiable at points of interaction with other shocks and points which are centers of centered compression waves. In other words, all the shocks curves are piecewise $C^{k+1}$ curves.

\section{Piecewise Smoothness}

In Section 2, we have studied the local structure of the solutions under the condition that $L_{3}=\emptyset$. We can deduce that the solutions are piecewise smooth based on the local structure of the solutions under the same condition. On the other hand we can obtain the asymptotical curves of the shock curves using the technique similar to the Lemma 4.1 of Schaeffer in [9], thus we can show there can be finitely many shock curves under certain conditions. Now we will show all of the results mentioned above in detail. There are only finitely many connected components of $\mathscr{M}$ for $F(x, t, \bullet)$ for each given point $(x, t) \in H$ since $L_{3}=\emptyset$, and so we can know easily $(x, t) \in U \cup \bar{\Gamma}_{1} \cup \bar{\Gamma}_{0}^{(f)} \cup \bar{\Gamma}_{0}^{(c)}$ for each $(x, t) \in \mathbb{R} \times(0, \infty)$ i.e. $H=U \cup \bar{\Gamma}_{1} \cup \bar{\Gamma}_{0}^{(f)} \cup \bar{\Gamma}_{0}^{(c)}$ provided that $L_{3}=\emptyset$. By Lemma 2.1, $\Gamma=\bar{\Gamma}_{1} \cup \bar{\Gamma}_{0}^{(f)} \cup \bar{\Gamma}_{0}^{(c)}$ is a closed subset of $H$, and $\Gamma$ is covered by neighborhoods of the type described in Theorem 2.1 and Theorem 2.2. For any compact set $K \subset H$, by choosing a finite subcover of $K \cap \Gamma$ we see that $K \cap \Gamma$ consists of the union of a finite number of shock and each shock is piecewise $C^{k+1}$ curve. Therefore the minimizing function $u(x, t)$ is piecewise $C^{k}$ smooth if $L_{3}=\emptyset$. It is worth pointing out that our piecewise smooth solution contain centered compression waves, which implies the class of piecewise smooth solutions with $C^{k}$ initial data except a first category subset $\Omega_{3}$ in this paper is wider than the class of the piecewise solutions with $C^{k}$ initial data, except a first category subset $\Omega_{2}$ considered by Dafermos in [2].

Dafermos [2] has proved that the initial data belonging to $\Omega_{2}$ is of first category. Thus $\Omega_{3}$ as a proper subset of $\Omega_{2}$ is of first category. Consequently we get the following theorem.

Theorem 3.1 There is a set $\Omega_{3} \subset C^{k}(\mathbb{R})$ of the first category such that for $\phi \in \mathscr{S}(\mathbb{R}) \sim \Omega_{3}$, $H=U \cup \bar{\Gamma}_{1} \cup \bar{\Gamma}_{0}^{(f)} \cup \bar{\Gamma}_{0}^{(c)}$, where $\Omega_{3}$ is a proper subset of $\Omega_{2}$. the solutions are piecewise smooth.

Next we show there are finite number of shocks under certain condition.

Li-Wang [6] in Theorem 4 in Section 3 and Theorem 5 in Section 4 have proved that there is an open dense set $\triangle \subset \mathscr{S}(\mathbb{R})$ such that for $\phi \in \triangle$, the associated function $\Phi$ attains its minimum 
over $\mathbb{R}$ only at points $a_{i}\left(\left|a_{i}\right|<\infty\right)$ and $\phi^{\left(l_{i}\right)}\left(a_{i}\right) \neq 0, \phi^{\left(l_{i}-1\right)}\left(a_{i}\right)=\cdots=\phi\left(a_{i}\right)=0$, where $l_{i}$ $(i=1, \cdots, m)$ is some integer, then for sufficiently large $t$ there are precisely $m+1$ smooth shocks. The proofs of them are similar to Lemma 4.1 and Proposition 4.2 in Section 4 in Schaeffer [9], so we omit them. Consequently we see there is an open dense set $\triangle \subset \mathscr{S}(\mathbb{R})$ such that for $\phi \in \triangle$, there are finitely many shock curves for sufficiently large $t$. Choose $T$ such that for $t>T$ there are only finitely many smooth shock curves. It is a simple to show that there is a constant $C$ such that no shocks form in the region $\{(x, t):|x| \geq C, 0 \leq t \leq T\}$. Of course only finitely many shocks form in the compact region $\{(x, t):|x| \leq C, 0 \leq t \leq T\}$, so we can get the following theorem:

Theorem 3.2 There is a set $\Omega_{4}=\Omega_{3} \cup \triangle^{c} \subset \mathscr{S}(\mathbb{R})$ of the first category such that for $\phi \in$ $\mathscr{S}(\mathbb{R}) \sim \Omega_{4}, H=U \cup \bar{\Gamma}_{1} \cup \bar{\Gamma}_{0}^{(f)} \cup \bar{\Gamma}_{0}^{(c)}$, the solutions of (1.1) are piecewise smooth and there are finite number of shocks, where $\triangle^{c}=\mathscr{S}(\mathbb{R}) \sim \triangle$.

\section{Concluding remarks}

In this work we proved that if the initial data do not belong to a very small subset of $C^{k}$ then the solutions of scalar conservation laws are piecewise smooth. It is important to understand the conditions under which the solution of the conservation law (1.1) is piecewise smooth, since most practical cases deal with the piecewise smooth solutions. For this reason, there have many studies on approximation methods for conservation laws whose solutions are piecewise smooth. For example, for systems of conservation laws, Goodman and Xin [3] proved that the viscosity methods approximating piecewise smooth solutions with finitely many noninteracting shocks have a local first-order rate of convergence away from the shocks; on the other hand, for scalar conservation laws, the global rate of convergence for the viscosity methods can be obtained [14, 15], and the point-wise rate of convergence for the viscosity methods has been obtained [12, 13].

In this work, we have introduced a new approach for studying the solution structures for the conservation laws, which is particularly suitable for handling the larger class of initial data considered in this works. We point out that Thom's theory of catastrophes [10], which plays a key role in Schaeffer [9], can not be used to analyze the larger class of initial data. The main motivation of this study is to develop a new analysis approach which can be extended to study the solution structures for the Hamilton-Jacobi equations. The study along this direction is under investigation, and some relevant results will be reported elsewhere.

\section{A Appendix}

Lemma A.1 Set $m(x, t)=\min _{u \in \mathbb{R}} F(x, t, u)$. Then $m(x, t)$ is a continuous function of $x, t$ in the semi-plane $t>0$.

Proof: For each fixed point $\left(x_{0}, t_{0}\right)$, let $m\left(x_{0}, t_{0}\right)=F\left(x_{0}, t_{0}, u_{0}\right)$ and $\left(x_{n}, t_{n}\right)$ with $n \geq 1$ be any sequence convergent to $\left(x_{0}, t_{0}\right)$. Now we prove $\lim _{n \rightarrow \infty} m\left(x_{n}, t_{n}\right)=m\left(x_{0}, t_{0}\right)$. In fact, letting 
$m\left(x_{n}, t_{n}\right)=F\left(x_{n}, t_{n}, u_{n}\right)$ gives

$$
\begin{aligned}
& m\left(x_{0}, t_{0}\right)-m\left(x_{n}, t_{n}\right) \leq F\left(x_{0}, t_{0}, u_{n}\right)-F\left(x_{n}, t_{n}, u_{n}\right) \\
= & t_{0} g\left(u_{n}\right)+\Phi\left(x_{0}-t_{0} a\left(u_{n}\right)\right)-t_{n} g\left(u_{n}\right)-\Phi\left(x_{n}-t_{n} a\left(u_{n}\right)\right) \\
= & \left(t_{0}-t_{n}\right) g\left(u_{n}\right)+\Phi\left(x_{0}-t_{0} a\left(u_{n}\right)\right)-\Phi\left(x_{n}-t_{n} a\left(u_{n}\right)\right) \\
= & \left(t_{0}-t_{n}\right) g\left(u_{n}\right)+\phi(\xi)\left[x_{0}-x_{n}+\left(t_{n}-t_{0}\right) a\left(u_{n}\right)\right] \\
\leq & M\left(\left|x_{n}-x_{0}\right|+\left|t_{n}-t_{0}\right|\right) \rightarrow 0, \quad \text { as } n \rightarrow \infty,
\end{aligned}
$$

where $\xi$ is between $x_{0}-t_{0} a\left(u_{n}\right)$ and $x_{n}-t_{n} a\left(u_{n}\right), M$ is a positive constant. On the other hand, since $F\left(x, t, u_{0}\right)$ is continuous at $\left(x_{0}, t_{0}\right)$ as $u_{0}$ is fixed,

$$
m\left(x_{0}, t_{0}\right)-m\left(x_{n}, t_{n}\right) \geq F\left(x_{0}, t_{0}, u_{0}\right)-F\left(x_{n}, t_{n}, u_{0}\right) \rightarrow 0, \text { as } n \rightarrow \infty .
$$

Combining (A.1) and (A.2), we have $m\left(x_{0}, t_{0}\right)-m\left(x_{n}, t_{n}\right) \rightarrow 0$, as $n \rightarrow \infty$. Thus $m(x, t)$ is continuous at $\left(x_{0}, t_{0}\right)$.

Lemma A.2 If $\left(x_{l}, t_{1}\right),\left(x_{r}, t_{1}\right) \in \Theta\left(t_{1}>t_{0}\right)$ are the intersection points of the line $t=t_{1}$ with the curves (2.19) and (2.20), then $x_{r}-x_{l}>0$.

Proof: It follows from (2.19) and (2.20) that there exist points $\bar{x}_{1} \in\left(y\left(x_{0}, t_{0}, \alpha\right), y\left(x_{0}, t_{0}, \alpha\right)+\delta_{0}\right)$ and $\bar{x}_{2} \in\left(y\left(x_{0}, t_{0}, \beta\right)-\delta_{0}, y\left(x_{0}, t_{0}, \beta\right)\right)$ such that $x_{l}=\bar{x}_{1}+t_{1} a\left(\phi\left(\bar{x}_{1}\right)\right)$ and $x_{r}=\overline{x_{2}}+t_{1} a\left(\phi\left(\overline{x_{2}}\right)\right)$. Consequently,

$$
\begin{aligned}
& x_{r}-x_{l}=x_{r}-x_{2}+x_{2}-x_{1}+x_{1}-x_{l} \\
& =\bar{x}_{2}+t_{1} a\left(\phi\left(\bar{x}_{2}\right)\right)-\left(y\left(x_{0}, t_{0}, \beta\right)+t_{1} a\left(\phi\left(y\left(x_{0}, t_{0}, \beta\right)\right)\right)\right) \\
& \quad+y\left(x_{0}, t_{0}, \beta\right)+t_{1} a\left(\phi\left(y\left(x_{0}, t_{0}, \beta\right)\right)\right)-\left(y\left(x_{0}, t_{0}, \alpha\right)+t_{1} a\left(\phi\left(y\left(x_{0}, t_{0}, \alpha\right)\right)\right)\right) \\
& \quad+y\left(x_{0}, t_{0}, \alpha\right)+t_{1} a\left(\phi\left(y\left(x_{0}, t_{0}, \alpha\right)\right)\right)-\left(\bar{x}_{1}+t_{1} a\left(\phi\left(\bar{x}_{1}\right)\right)\right),
\end{aligned}
$$

where $\left.x_{2}=y\left(x_{0}, t_{0}, \beta\right)\right)+t_{1} a\left(\phi\left(y\left(x_{0}, t_{0}, \beta\right)\right)\right)$ and $\left.x_{1}=y\left(x_{0}, t_{0}, \beta\right)\right)+t_{1} a\left(\phi\left(y\left(x_{0}, t_{0}, \beta\right)\right)\right)$. Observe

$$
\begin{aligned}
& x_{2}-x_{1} \\
= & y\left(x_{0}, t_{0}, \beta\right)+t_{1} a\left(\phi\left(y\left(x_{0}, t_{0}, \beta\right)\right)\right)-\left(y\left(x_{0}, t_{0}, \alpha\right)+t_{1} a\left(\phi\left(y\left(x_{0}, t_{0}, \alpha\right)\right)\right)\right) \\
= & y\left(x_{0}, t_{0}, \beta\right)-y\left(x_{0}, t_{0}, \alpha\right)+t_{1}\left(a\left(\phi\left(y\left(x_{0}, t_{0}, \beta\right)\right)\right)-a\left(\phi\left(y\left(x_{0}, t_{0}, \alpha\right)\right)\right)\right) \\
= & y\left(x_{0}, t_{0}, \beta\right)-y\left(x_{0}, t_{0}, \alpha\right)+t_{1}(a(\phi(\xi)))^{\prime}\left(y\left(x_{0}, t_{0}, \beta\right)-y\left(x_{0}, t_{0}, \alpha\right)\right) \\
= & \left(y\left(x_{0}, t_{0}, \beta\right)-y\left(x_{0}, t_{0}, \alpha\right)\right)\left[1+t_{1}\left(-\frac{1}{t_{0}}\right)\right] \geq 0,
\end{aligned}
$$

where $y\left(x_{0}, t_{0}, \beta\right)<\xi<y\left(x_{0}, t_{0}, \alpha\right)$. Moreover,

$$
\begin{aligned}
& x_{r}-x_{2} \\
= & \bar{x}_{2}+t_{1} a\left(\phi\left(\bar{x}_{2}\right)\right)-\left(y\left(x_{0}, t_{0}, \beta\right)+t_{1} a\left(\phi\left(y\left(x_{0}, t_{0}, \beta\right)\right)\right)\right) \\
= & \bar{x}_{2}-y\left(x_{0}, t_{0}, \beta\right)-\frac{1}{\left(a\left(\phi\left(\bar{x}_{2}\right)\right)\right)^{\prime}}\left[a\left(\phi\left(\bar{x}_{2}\right)\right)-a\left(\phi\left(y\left(x_{0}, t_{0}, \beta\right)\right)\right)\right] \\
= & \bar{x}_{2}-y\left(x_{0}, t_{0}, \beta\right)+\frac{1}{\left(a\left(\phi\left(\bar{x}_{2}\right)\right)\right)^{\prime}}\left[\left(a\left(\phi\left(\bar{x}_{2}\right)\right)\right)^{\prime}\left(y\left(x_{0}, t_{0}, \beta\right)-\bar{x}_{2}\right)\right. \\
& \left.\quad+\left(y\left(x_{0}, t_{0}, \beta\right)-\bar{x}_{2}\right)^{2} \int_{0}^{1}(1-t)(a(\phi))^{\prime \prime}\left(\bar{x}_{2}+t\left(y\left(x_{0}, t_{0}, \beta\right)-\bar{x}_{2}\right)\right) d t\right] \\
& \left.\quad \frac{1}{\left(a\left(\phi\left(\bar{x}_{2}\right)\right)\right)^{\prime}}\left(y\left(x_{0}, t_{0}, \beta\right)-\bar{x}_{2}\right)^{2} \int_{0}^{1}(1-t)(a(\phi))^{\prime \prime}\left(\bar{x}_{2}+t\left(y\left(x_{0}, t_{0}, \beta\right)-\bar{x}_{2}\right)\right) d t\right]>0,
\end{aligned}
$$


for each $\bar{x}_{2} \in\left(y\left(x_{0}, t_{0}, \beta\right)-\delta_{0}, y\left(x_{0}, t_{0}, \beta\right)\right)$, where we have used the fact $\left(a\left(\phi\left(\bar{x}_{2}\right)\right)\right)^{\prime}<0,(2.13)$ and (2.16). Similarly, we have $x_{1}-x_{l}>0$. It follows from (A.3)-(A.5) that $x_{r}-x_{l}=x_{r}-x_{2}+$ $x_{2}-x_{1}+x_{1}-x_{l}>0$.

Lemma A.3 Suppose $L_{3}=\emptyset$. If $\left[u_{1}^{-}, u_{1}^{+}\right]$and $\left[u_{2}^{-}, u_{2}^{+}\right]$are two neighboring connected components of $\mathscr{M}_{\left(x_{1}, t_{1}\right)}$, where $\left.u_{1}^{-} \leq u_{1}^{+}<u_{2}^{-} \leq u_{2}^{+}\right]$, then there exists an open neighborhood $\bar{\Theta}$ of $\left(x_{1}, t_{1}\right)$ such that $\bar{\Theta} \cap G \cap \Gamma_{1}$ contains a half curve $x=\gamma^{-}(t)$ terminating at $\left(x_{1}, t_{1}\right)$, where $G$ is the triangle domain formed by $t=0$, the characteristics $x=y\left(x_{1}, t_{1}, u_{2}^{-}\right)+t a\left(u_{2}^{-}\right)$and $x=y\left(x_{1}, t_{1}, u_{1}^{+}\right)+$ $\operatorname{ta}\left(u_{1}^{+}\right)$.

Proof: By an argument similar to the proof of assertion (2.18), there exists a neighborhood $\bar{\Theta}$ of $\left(x_{1}, t_{1}\right)$ and two intervals $\left(y\left(x_{1}, t_{1}, u_{1}^{+}\right)-\delta, y\left(x_{1}, t_{1}, u_{1}^{+}\right)\right)$and $\left(y\left(x_{1}, t_{1}, u_{2}^{-}\right), y\left(x_{1}, t_{1}, u_{2}^{-}\right)+\delta\right)$ such that for each given $(x, t) \in \bar{\Theta}^{\prime} \cap G$ and any minimizing value, say $u$, for $F(x, t, \bullet), y(x, t, u)$ belongs to $\left(y\left(x_{1}, t_{1}, u_{1}^{+}\right)-\delta, y\left(x_{1}, t_{1}, u_{1}^{+}\right)\right)$or $\left(y\left(x_{1}, t_{1}, u_{2}^{-}\right), y\left(x_{1}, t_{1}, u_{2}^{-}\right)+\delta\right)$. Now we claim that: for each $(x, t) \in \bar{\Theta}^{\prime} \cap G$ there exists a unique minimizing value $u(x, t)$ for $F(x, t, \bullet)$ such that $y(x, t, u(x, t)) \in$ $\left(y\left(x_{1}, t_{1}, u_{1}^{+}\right)-\delta, y\left(x_{1}, t_{1}, u_{1}^{+}\right)\right)$and it is non-degenerate, where $\bar{\Theta}^{\prime}=\bar{\Theta} \sim\left\{\left(x_{1}, t_{1}\right)\right\}$. In fact, there are only two cases to be considered

Case 1: $F_{u u}\left(x_{1}, t_{1}, u_{1}^{+}\right)=0$. In this case, if there exist a point $(\tilde{x}, \tilde{t}) \in \bar{\Theta}^{\prime} \cap G$ such that there exist at least two minimizing values $u^{*}$ and $u^{* *}$ for $F(\tilde{x}, \tilde{t}, \bullet)$ and $y\left(\tilde{x}, \tilde{t}, u^{*}\right), y\left(\tilde{x}, \tilde{t}, u^{* *}\right) \in$ $\left(y\left(x_{1}, t_{1}, u_{1}^{+}\right)-\delta, y\left(x_{1}, t_{1}, u_{1}^{+}\right)\right)$. Thus there exist at least two characteristics passing through $(\tilde{x}, \tilde{t})$ :

$$
\begin{aligned}
& x=y\left(\tilde{x}, \tilde{t}, u^{*}\right)+\operatorname{ta}\left(u^{*}\right), \\
& x=y\left(\tilde{x}, \tilde{t}, u^{* *}\right)+\operatorname{ta}\left(u^{* *}\right) .
\end{aligned}
$$

On the other hand, by using the fact that $(a(\phi(x)))^{\prime}$ is monotone decreasing on the interval $\left(y\left(x_{1}, t_{1}, u_{1}^{+}\right)-\delta, y\left(x_{1}, t_{1}, u_{1}^{+}\right)\right)$and the assertion $(2.16)$, we have

$$
\tilde{t}=-\frac{y\left(\tilde{x}, \tilde{t}, u^{*}\right)-y\left(\tilde{x}, \tilde{t}, u^{* *}\right)}{a\left(u^{*}\right)-a\left(u^{* *}\right)}=-\frac{1}{(a(\phi(\eta)))^{\prime}}>t_{1},
$$

where $\eta$ is between $y\left(\tilde{x}, \tilde{t}, u^{*}\right)$ and $y\left(\tilde{x}, \tilde{t}, u^{* *}\right)$. This result is contradictory to the fact that $\tilde{t}<t_{1}$. The claim is therefore proved.

Case 2: $F_{u u}\left(x_{1}, t_{1}, u_{1}^{+}\right) \neq 0$. In this case, since $F_{u u}\left(x_{1}, t_{1}, u_{1}^{+}\right) \neq 0$, it follows from the Implicit Function Theorem that there is an open neighborhood $U\left(u_{1}^{+}\right)$of $u_{1}$ such that for $(x, t)$ close to $\left(x_{1}, t_{1}\right)$ the equation $(\partial F / \partial u)(x, t, u)=0$ has a unique solution $u(x, t) \in U\left(u_{1}^{+}\right)$. Consequently, there is a unique minimizing value $u(x, t)$ such that $y(x, t, u(x, t)) \in\left(y\left(x_{1}, t_{1}, u_{1}^{+}\right)-\delta, y\left(x_{1}, t_{1}, u_{1}^{+}\right)\right)$.

Similarly we can deduce that There exists a unique minimizing value $u(x, t)$ for $F(x, t, \bullet)$ for each $(x, t) \in \bar{\Theta}^{\prime} \cap G$, such that $y(x, t, u(x, t)) \in\left(y\left(x_{1}, t_{1}, u_{2}^{-}\right), y\left(x_{1}, t_{1}, u_{2}^{-}\right)+\delta\right)$ and it is non-degenerate.

Let $A_{t_{2}}=\left\{x \mid\left(x, t_{2}\right) \in \bar{\Theta}^{\prime} \cap G\right\}$. There exists a characteristic which originates from a point on the left of the point $\left(y\left(x_{1}, t_{1}, u_{1}^{+}\right), 0\right)$ and passes through $\left.\left(x_{1}, t_{2}\right)\right\}$. Let $B_{t_{2}}=\left\{x \mid\left(x, t_{2}\right) \in\right.$ $\left.\bar{\Theta}^{\prime} \cap G\right\}$. There exists a characteristic which originates from a point on the right of the point $\left(y\left(x_{1}, t_{1}, u_{2}^{-}\right), 0\right)$ and passes through $\left.\left(x_{1}, t_{2}\right)\right\}$. Let $\bar{x}_{1}$ be the infimum of the set $A_{t_{2}}$ and $\bar{x}_{2}$ be the supremum of the set $B_{t_{2}}$. By an argument similar to the proofs of the assertion (2.35) and (2.36), it can be shown that there exist two minimizing values for $F\left(\bar{x}_{1}, t_{2}, \bullet\right)$ which are non-degenerate. 
This implies there are two valid characteristics passing through $\left(\bar{x}_{1}, t_{2}\right)$, one originated from a point on the left of the point $\left(y\left(x_{1}, t_{1}, u_{1}^{+}\right), 0\right)$ and the other originated from a point on the right of the point $\left(y\left(x_{1}, t_{1}, u_{2}^{-}\right), 0\right)$. Similarly, it can be shown that there exist two minimizing values for $F\left(\bar{x}_{2}, t_{2}, \bullet\right)$ which are non-degenerate. This implies that there are two valid characteristics passing through $\left(\bar{x}_{2}, t_{2}\right)$, one originated from a point on the left of the point $\left(y\left(x_{1}, t_{1}, u_{1}^{+}\right), 0\right)$ and the other originated from a point on the right of the point $\left(y\left(x_{1}, t_{1}, u_{2}^{-}\right), 0\right)$. By the same argument for obtaining the assertion (2.37) we have

$$
\bar{x}_{1}=\bar{x}_{2}
$$

Consequently, a unique half-curve terminating at $\left(x_{1}, t_{1}\right)$ denoted by $x=\gamma^{-}(t)$ is defined for $t \leq t_{1}$. Thus we complete the proof.

Lemma A.4 The curve (2.19) (respective (2.20)) is continuous and is the closure of union of countable open arcs, on which the curve is $C^{k}$. The points at which the curve (2.19) (respective (2.20))is not differentiable belong to the end points of the open arcs but the left derivative continuous on the left and right derivative continuous on the right.

Proof: In fact, it is easy to compute that

$$
x^{\prime}(\xi)=\frac{(a(\phi(\xi)))^{\prime}(a(\phi(\xi)))^{\prime \prime}}{(a(\phi(\xi)))^{\prime 2}}, \quad t^{\prime}(\xi)=\frac{(a(\phi(\xi)))^{\prime \prime}}{(a(\phi(\xi)))^{\prime 2}},
$$

for $\xi \in\left[y\left(x_{0}, t_{0}, \alpha\right), y\left(x_{0}, t_{0}, \alpha\right)+\delta_{0}\right]$. Let

$$
A_{t}=\{\xi \mid t(\xi)=t\}, \quad A_{x}=\{\xi \mid x(\xi)=x\},
$$

where $x$ and $t$ are given. Obviously, $A_{t}$ is closed. Since $L_{3}=\emptyset$, we have $(a(\phi(\xi)))^{\prime \prime} \geq 0$ for each $\xi \in\left[y\left(x_{0}, t_{0}, \alpha\right), y\left(x_{0}, t_{0}, \alpha\right)+\delta_{0}\right]$. We first prove

$$
x(\xi) \text { is a function of } t(\xi) .
$$

For any fixed $t_{1}=t(\xi)$ with $\xi \in A_{t_{1}}$, there exists a unique $x_{1}(\xi)$ to $t_{1}$. In fact, if $A_{t_{1}}=\left\{\xi_{1}\right\}$, we have $x_{1}=x\left(\xi_{1}\right)$ which is unique. If $A_{t_{1}}=\left[\alpha_{1}, \beta_{1}\right], \alpha_{1} \neq \beta_{1}$, we have $t^{\prime}(\xi)=0$, which implies $x^{\prime}(\xi)=0$ in light of (A.9), for each $\xi \in\left[\alpha_{1}, \beta_{1}\right]$. Consequently, $t\left(\xi_{1}\right)=t\left(\xi_{2}\right)$ implies $x\left(\xi_{1}\right)=t\left(\xi_{2}\right)$, here $\xi_{1}, \xi_{2} \in\left[\alpha_{1}, \beta_{1}\right]$. Then $x_{1}(\xi)$ is unique. This completes the proof of assertion (A.11). The curve (2.19) can be denoted by $x=\beta(t)$. Next we claim

$$
x=\beta(t) \text { is a continuous function of } t .
$$

For each fixed point $t_{1}$, we only need to prove

$$
\lim _{t \rightarrow t_{1}+} \beta(t)=\lim _{t \rightarrow t_{1}-} \beta(t)=\beta\left(t_{1}\right) .
$$

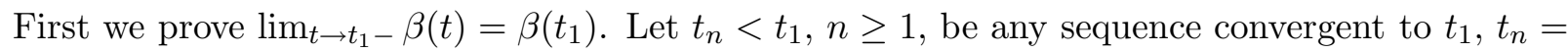
$t\left(\xi_{n}\right)$ and $t_{1}=t\left(\xi_{1}\right)$, where $\xi_{n}=\sup A_{t_{n}}$ and $\xi_{1}=\inf A_{t_{1}}$. We will show $\lim _{n \rightarrow \infty} \beta\left(t_{n}\right)=\beta\left(t_{1}\right)$. Notice the function $t$ is an increasing function of $\xi$, we have $\lim _{n \rightarrow \infty} \xi_{n}=\xi_{1}$. Consequently,

$$
\lim _{n \rightarrow \infty} \beta\left(t_{n}\right)=\lim _{n \rightarrow \infty} \beta\left(t\left(\xi_{n}\right)\right)=\lim _{n \rightarrow \infty} x\left(\xi_{n}\right)=x\left(\xi_{1}\right)=\beta\left(t_{1}\right) .
$$


Then $\lim _{t \rightarrow t_{1}-} \beta(t)=\beta\left(t_{1}\right)$. Similarly, we have $\lim _{t \rightarrow t_{1}+} \beta(t)=\beta\left(t_{1}\right)$. So the assertion (A.12) is true. Finally we prove

$$
x=\beta(t) \text { is differentiable at point } t_{1} \Longleftrightarrow \text { the set } A_{t_{1}} \text { contains only one point. }
$$

Let $A_{t_{1}}=\left[\xi_{1}, \xi_{2}\right]$ and $t_{n}<t_{1}, n \geq 1$, be any sequence convergent to $t_{1}, t_{n}=t\left(\xi_{n}\right)$ and $t_{1}=t\left(\xi_{1}\right)$, with $\xi_{n}=\sup A_{t_{n}}$ and $\xi_{1}=\inf A_{t_{1}}$. Observe

$$
\begin{aligned}
& \frac{\beta\left(t_{n}\right)-\beta\left(t_{1}\right)}{t_{n}-t_{1}}=\frac{x\left(\xi_{n}\right)-x\left(\xi_{1}\right)}{t\left(\xi_{n}\right)-t\left(\xi_{1}\right)} \\
= & \frac{\xi_{n}-a\left(\phi\left(\xi_{n}\right)\right) /\left(a\left(\phi\left(\xi_{n}\right)\right)\right)^{\prime}-\xi_{1}+a\left(\phi\left(\xi_{1}\right)\right) /\left(a\left(\phi\left(\xi_{1}\right)\right)\right)^{\prime}}{1 /\left(a\left(\phi\left(\xi_{1}\right)\right)\right)^{\prime}-1 /\left(a\left(\phi\left(\xi_{n}\right)\right)\right)^{\prime}} \\
= & a\left(\phi\left(\xi_{1}\right)\right)+T_{1},
\end{aligned}
$$

where the last term is non-negative:

$$
\begin{aligned}
T_{1} & :=\frac{\xi_{n}-\xi_{1}+\left(a\left(\phi\left(\xi_{1}\right)\right)-a\left(\phi\left(\xi_{n}\right)\right)\right) /\left(a\left(\phi\left(\xi_{n}\right)\right)\right)^{\prime}}{1 /\left(a\left(\phi\left(\xi_{1}\right)\right)\right)^{\prime}-1 /\left(a\left(\phi\left(\xi_{n}\right)\right)\right)^{\prime}} \\
& =\left(a\left(\phi\left(\xi_{n}\right)\right)\right)^{\prime}\left(a\left(\phi\left(\xi_{1}\right)\right)\right)^{\prime} \frac{\xi_{n}-\xi_{1}+\left(a\left(\phi\left(\xi_{1}\right)\right)-a\left(\phi\left(\xi_{n}\right)\right)\right) /\left(a\left(\phi\left(\xi_{n}\right)\right)\right)^{\prime}}{\left(a\left(\phi\left(\xi_{n}\right)\right)\right)^{\prime}-\left(a\left(\phi\left(\xi_{1}\right)\right)\right)^{\prime}} \\
& =\left(a\left(\phi\left(\xi_{n}\right)\right)\right)^{\prime}\left(a\left(\phi\left(\xi_{1}\right)\right)\right)^{\prime} \frac{(a(\phi(\tilde{\xi})))^{\prime \prime}\left(\xi_{n}-\xi_{1}\right)^{2}}{2\left(a\left(\phi\left(\xi_{n}\right)\right)\right)^{\prime}\left[\left(a\left(\phi\left(\xi_{n}\right)\right)\right)^{\prime}-\left(a\left(\phi\left(\xi_{1}\right)\right)\right)^{\prime}\right]} \geq 0
\end{aligned}
$$

where $\tilde{\xi}$ lies between $\xi_{n}$ and $\xi_{1}$ and satisfies

$$
a\left(\phi\left(\xi_{1}\right)\right)-a\left(\phi\left(\xi_{n}\right)\right)=\left(a\left(\phi\left(\xi_{n}\right)\right)\right)^{\prime}\left(\xi_{1}-\xi_{n}\right)+\frac{1}{2 !}(a(\phi(\tilde{\xi})))^{\prime \prime}\left(\xi_{1}-\xi_{n}\right)^{2} .
$$

In the last step of (A.15), we have used the facts that $(a(\phi(\tilde{\xi})))^{\prime \prime} \geq 0$ and $\left(a\left(\phi\left(\xi_{n}\right)\right)\right)^{\prime}-\left(a\left(\phi\left(\xi_{1}\right)\right)\right)^{\prime}<$ 0 , which are due to the monotonicity of $(a(\phi(\xi)))^{\prime}$, the assertion $(2.17)$ and $\xi_{n}<\xi_{1}$. Similarly,

$$
\frac{\beta\left(t_{n}\right)-\beta\left(t_{1}\right)}{t_{n}-t_{1}}=a\left(\phi\left(\xi_{n}\right)\right)+T_{2}
$$

where

$$
\begin{aligned}
T_{2} & :=\frac{\xi_{n}-\xi_{1}+\left(a\left(\phi\left(\xi_{1}\right)\right)-a\left(\phi\left(\xi_{n}\right)\right)\right) /\left(a\left(\phi\left(\xi_{1}\right)\right)\right)^{\prime}}{1 /\left(a\left(\phi\left(\xi_{1}\right)\right)\right)^{\prime}-1 /\left(a\left(\phi\left(\xi_{n}\right)\right)\right)^{\prime}} \\
& =-\left(a\left(\phi\left(\xi_{n}\right)\right)\right)^{\prime}\left(a\left(\phi\left(\xi_{1}\right)\right)\right)^{\prime} \frac{(a(\phi(\hat{\xi})))^{\prime \prime}\left(\xi_{n}-\xi_{1}\right)^{2}}{2\left(a\left(\phi\left(\xi_{1}\right)\right)\right)^{\prime}\left[\left(a\left(\phi\left(\xi_{n}\right)\right)\right)^{\prime}-\left(a\left(\phi\left(\xi_{1}\right)\right)\right)^{\prime}\right]} \leq 0
\end{aligned}
$$

where $\hat{\xi}$ is between $\xi_{n}$ and $\xi_{1}$ and satisfies

$$
a\left(\phi\left(\xi_{n}\right)\right)-a\left(\phi\left(\xi_{1}\right)\right)=\left(a\left(\phi\left(\xi_{1}\right)\right)\right)^{\prime}\left(\xi_{n}-\xi_{1}\right)+\frac{1}{2 !}(a(\phi(\hat{\xi})))^{\prime \prime}\left(\xi_{n}-\xi_{1}\right)^{2} .
$$

Combining (A.14)-(A.17) gives

$$
a\left(\phi\left(\xi_{1}\right)\right) \leq \frac{\beta\left(t_{n}\right)-\beta\left(t_{1}\right)}{t_{n}-t_{1}} \leq a\left(\phi\left(\xi_{n}\right)\right)
$$


Consequently,

$$
\lim _{t_{n} \rightarrow t_{1}-} \frac{\beta\left(t_{n}\right)-\beta\left(t_{1}\right)}{t_{n}-t_{1}}=a\left(\phi\left(\xi_{1}\right)\right)
$$

which implies that

$$
\lim _{t \rightarrow t_{1}-} \frac{\beta(t)-\beta\left(t_{1}\right)}{t-t_{1}}=a\left(\phi\left(\xi_{1}\right)\right)
$$

Similarly,

$$
\lim _{t \rightarrow t_{1}+} \frac{\beta(t)-\beta\left(t_{1}\right)}{t-t_{1}}=a\left(\phi\left(\xi_{2}\right)\right) .
$$

The above two results yield $\beta_{-}^{\prime}\left(t_{1}\right)=a\left(\phi\left(\xi_{1}\right)\right)$ and $\beta_{+}^{\prime}\left(t_{1}\right)=a\left(\phi\left(\xi_{2}\right)\right)$. Thus the function $\beta(t)$ is differentiable at $t_{1}$ if and only if $\xi_{1}=\xi_{2}$. This verifies the assertion (A.13). It is easy to show that there are countable points $(\beta(t), t)$ such that the set $A_{t}$ contains more than one point. Moreover, it can be shown that the set

$$
\left\{(\beta(t), t) \mid A_{t}=\{\xi\},(a(\phi(\xi)))^{\prime \prime} \neq 0\right\}
$$

is an open set, and is therefore a union of disjoint open arcs. On the other hand, the set

$$
\left\{(\beta(t), t) \mid A_{t}=\left[\xi_{1}, \xi_{2}\right],\left(a\left(\phi\left(\xi_{1}\right)\right)\right)^{\prime \prime}=0\right\}
$$

is a closed set and contains at most countable points. Each point belonging to the set (A.23) is an end point of some open arcs of the set (A.22). This implies that the curve (2.19) (respective (2.20)) is continuous and is the closure of union of countable open arcs, on which the curve is $C^{k}$. The points at which the curve (2.19) (respective (2.20)) is not differentiable belong to the set (A.23); and the left derivative is continuous on the left and the right derivative is continuous on the right.

Acknowledgment. The research of this project was partially supported by CERG Grants of Hong Kong Research Grant Council, FRG grants of Hong Kong Baptist University, and International Research Team of Complex Systems of Chinese Academy of Sciences. The second author was supported by National Natural Foundation of China under contract 10371124.

\section{References}

[1] S. Chen And Z. Zhang, Formation of shock for first order quasilinear equations, Acta Sci. Nat. Univ. Fudan, 8 (1963), 13-22 (in Chinese).

[2] C.M. Dafermos, Characteristics in hyperbolic conservation laws, Nonlinear Analysis and Mathematics Heriot-Watt Symposium, Vol 1, 5-59, ed. R.J. Knops. London, Pitman, 1974.

[3] J. Goodman and Z. Xin, Viscous limits for piecewise smooth solutions to systems of conservation laws, Arch. Rational Mech. Anal. 121 (1992), pp. 235-265. 
[4] E. Hopf, The partial differential equation $u_{t}+u u_{x}=\mu u_{x x}$, Comm. Pure. Appl. Math. (1950), 201-230.

[5] P.D. Lax, Hyperbolic systems of conservation laws II, Comm. Pure. Appl. Math. 10 (1957), 537-566.

[6] B. Li And J. WAng, The global qualitative study of solutions to a conservation law, Sci. Special Math. Issue (1979), 12-24 (in Chinese).

[7] B. Li And J. WANG, The global qualitative study of solutions to a conservation law (II), Sci. Special Math. Issue (1979), 25-38 (in Chinese).

[8] O. A. Oleinik, Discontinuous solutions of nonlinear differential equations, in "Amer. Math. Soc. Transl.," Vol 26,pp.95-172, Amer. Math. Soc., Providence, 1963

[9] D.G. Schaeffer, A regularity theorem for conservation laws, Adv. Math. 11 (1973), 368386

[10] R. Thом, Stabilité Structurelle et Morphogenèse, Addison-Wesley, Reading, Massachusetts, 1972

[11] E. TAdmor And T. TASsa, On the piecewise smoothness of entropy solutions to scalar conservation laws, Commun. in PDEs 18 (1993), pp. 1631-1652.

[12] E. TAdmor and T. TAng, Pointwise convergence rate for scalar conservation laws with piecewise smooth solutions, SIAM J. Numer. Anal. 36 (1999) 1739-1758.

[13] E. TADmor And T. TAng, Pointwise error estimates for relaxation approximations to conservation laws, SIAM J. Math. Anal. 32 (2001), 870-886.

[14] T. Tang And Z.-H. Teng, Viscosity methods for piecewise smooth solutions to scalar conservation laws, Math. Comp. 66 (1997), pp. 495-526.

[15] T. TAng, Z.-H. Teng And Z.-P. XIn, Fractional rate of convergence for viscous approximation to nonconvex conservation laws, SIAM J. Math. Anal. 35 (2003), pp. 98-122. 